ESAIM: M2AN 48 (2014) 1583-1613

DOI: $10.1051 / \mathrm{m} 2 \mathrm{an} / 2014011$
ESAIM: Mathematical Modelling and Numerical Analysis

www.esaim-m2an.org

\title{
DERIVATION OF A HOMOGENIZED TWO-TEMPERATURE MODEL FROM THE HEAT EQUATION
}

\author{
Laurent Desvillettes ${ }^{1}$, François Golse ${ }^{2}$ and Valeria Ricci ${ }^{3}$
}

\begin{abstract}
This work studies the heat equation in a two-phase material with spherical inclusions. Under some appropriate scaling on the size, volume fraction and heat capacity of the inclusions, we derive a coupled system of partial differential equations governing the evolution of the temperature of each phase at a macroscopic level of description. The coupling terms describing the exchange of heat between the phases are obtained by using homogenization techniques originating from [D. Cioranescu, F. Murat, Collège de France Seminar, vol. II. Paris 1979-1980; vol. 60 of Res. Notes Math. Pitman, Boston, London (1982) 98-138].
\end{abstract}

Mathematics Subject Classification. 35K05, 35B27, 76T05, 35Q79, 76M50.

Received September 25, 2013. Revised February 4, 2014.

Published online September 9, 2014.

\section{DESCRIPTION OF THE PROBLEM}

\subsection{The homogenized two-temperature model}

The purpose of this paper is to derive a model governing the exchange of heat in a composite medium consisting of a background material with very small spherical inclusions of another material with large thermal conductivity. Specifically, we assume that the volume fraction of the inclusions is negligible, while the heat capacity of each inclusion is large.

Under some appropriate scaling assumptions on the size, volume fraction and heat capacity of the inclusions, the temperature field $T \equiv T(t, x)$ of the background material and the temperature field $\theta \equiv \theta(t, x)$ of the dispersed phase (i.e. the inclusions) satisfy

$$
\left\{\begin{array}{l}
\partial_{t} T-d \Delta_{x} T+4 \pi \varrho d(T-\theta)=0, \\
\frac{d}{d^{\prime}} \partial_{t} \theta+4 \pi \varrho d(\theta-T)=0
\end{array}\right.
$$

Keywords and phrases. Heat equation, homogenization, infinite diffusion limit, thermal nonequilibrium models.

1 Ecole Normale Supérieure de Cachan, CMLA, 61 Av. du Pdt. Wilson, 94235 Cachan cedex, France. desville@cmla.ens-cachan.fr

2 Ecole Polytechnique, Centre de Mathématiques L. Schwartz, 91128 Palaiseau cedex, France.

francois.golse@math.polytechnique.fr

3 Dipartimento di Matematica e Informatica, Università degli Studi di Palermo, Via Archirafi 34, 90123 Palermo, Italy. valeria.ricci@unipa.it 
where $\varrho \equiv \varrho(x)$ is the number density of inclusions while $d$ and $d^{\prime}$ are the heat diffusion coefficients (i.e. the ratio of the heat conductivity to the volumetric heat capacity) of the background material and the inclusions, respectively.

Our work is motivated by a class of models used in the theory of multiphase flows, especially of multiphase flows in porous media. In such flows, each phase can have its own temperature (in which case the flow is said to be in thermal local non-equilibrium). Averaged equations for those temperatures similar to (1.1) have been proposed in $[6,10,15]$ on the basis of arguments at a macroscopic level of description. While these references address the case of complex realistic flows, our setting is purposedly chosen as simple as possible. Neither convection nor phase changes are taken into account in our model. Besides we only consider two phase flows, with only one phase having a positive diffusion rate. The case of positive diffusion rates is considered in ([15], Eqs. (13)-(15) on p. 242) and in ([10], on p. 2151), while the case of phases with zero diffusion rate is considered in ([6], Eqs. (1.63) and (1.74) on p. 39).

We give a rigorous derivation of the coupled system above from a model where the heat conductivity of the dispersed phase is assumed to be infinite from the outset. Our derivation is based on homogenization arguments following our earlier work in [9], inspired from $[8,11]$. The homogenization of the infinite conductivity problem in the case of a random distribution of inclusions has been obtained earlier in [14], assuming that the distribution of ball centers is given by an ergodic, stationary random process. The present work assumes very little information on the distribution of ball centers - apart from the separation hypothesis see (2.17) and (2.18) below.

For the sake of being complete, we also give a rigorous derivation of the infinite conductivity model from the classical heat diffusion equation. In the next two sections, we briefly describe the heat diffusion problem in a binary composite material, and the infinite conductivity model that is our starting point for the homogenization process.

Finally, we mention an earlier derivation [2] of the coupled system (1.1) as a homogenization limit - see also [13]. At variance with the present work, the reference [2] assumes a periodic distribution of inclusions with finite heat conductivity scaled to infinity in the homogenization limit. The periodicity assumption is not always relevant in the context of multiphase flows, and is systematically avoided in our work. In addition, the model with infinite heat conductivity considered as the starting point in our analysis cannot be reduced to a parabolic problem with discontinuous coefficients as in [2]. Together with the nonperiodic microstructure of the material, the particular nature of the transmission condition at the surface of inclusions with infinite heat conductivity accounts for significant differences with the analysis in [2]. The analysis in [2] has been extended in [3] to the case of inclusions of arbitrary shape, and to the wave equation of linear elasticity. We also mention [4], where the techniques used in [3] have been recently adapted to treat one case of nonperiodic fibered microstructures in a nonlinear elliptic model.

\subsection{The model with finite conductivity}

Consider a bounded open set $\Omega \subset \mathbf{R}^{3}$, let $A$ be an open subset of $\Omega$ and let $B=\Omega \backslash A$ be closed in $\mathbf{R}^{3}$. Assume that $\partial \Omega$ and $\partial B$ are submanifolds of $\mathbf{R}^{3}$ of class $C^{2}$, and that $B \cap \partial \Omega=\varnothing$. The unit normal field on the boundary of $B$ is oriented towards $A$.

The set $A$ is occupied by a material $\mathcal{A}$ with heat conductivity $\kappa_{A}$, density $\rho_{A}$ and specific heat capacity $C_{A}$, while the set $B$ is occupied by a material $\mathcal{B}$ with heat conductivity $\kappa_{B}$, density $\rho_{B}$ and specific heat capacity $C_{B}$. It will be assumed that $\rho_{A}, C_{A}, \kappa_{A}, \rho_{B}, C_{B}, \kappa_{B}$ are continuous positive functions on $\bar{A}$ and $B$, respectively. Denote by $T_{A}:=T_{A}(t, x)>0$ and $T_{B}:=T_{B}(t, x)>0$ the temperatures of $\mathcal{A}$ and $\mathcal{B}$ at time $t>0$ and point $x \in A(x \in B$, respectively).

Assuming that Fourier's law holds in both materials and that $T_{A}$ and $T_{B}$ are smooth (at least of class $C^{2}$ ) one has

$$
\begin{array}{ll}
\rho_{A}(x) C_{A}(x) \partial_{t} T_{A}(t, x)=\operatorname{div}_{x}\left(\kappa_{A}(x) \nabla_{x} T_{A}(t, x)\right), & x \in A, t>0, \\
\rho_{B}(x) C_{B}(x) \partial_{t} T_{B}(t, x)=\operatorname{div}_{x}\left(\kappa_{B}(x) \nabla_{x} T_{B}(t, x)\right), & x \in \stackrel{\circ}{B}, t>0 .
\end{array}
$$


If there is no heat source concentrated on the interface $\partial B$, then the temperature varies continuously across the interface between material $\mathcal{A}$ and material $\mathcal{B}$ and there is no net heat flux across that same interface. In other words, assuming that $T_{A}$ and $T_{B}$ are smooth up to the interface $\partial B$ between both materials

$$
\begin{cases}T_{A}(t, x)=T_{B}(t, x), & x \in \partial B, t>0, \\ \kappa_{A}(x) \frac{\partial T_{A}}{\partial n}(t, x)=\kappa_{B}(x) \frac{\partial T_{B}}{\partial n}(t, x), & x \in \partial B, t>0 .\end{cases}
$$

Define

$$
\rho(x):=\left\{\begin{array}{ll}
\rho_{A}(x) & x \in A \\
\rho_{B}(x) & x \in B
\end{array} \quad C(x):= \begin{cases}C_{A}(x) & x \in A \\
C_{B}(x) & x \in B\end{cases}\right.
$$

together with

$$
\kappa(x):= \begin{cases}\kappa_{A}(x) & x \in A \\ \kappa_{B}(x) & x \in B\end{cases}
$$

and

$$
T(t, x):= \begin{cases}T_{A}(t, x) & x \in A \\ T_{B}(t, x) & x \in B\end{cases}
$$

Assume that

$$
\left\{\begin{array}{l}
T_{A} \in C\left([0, \tau] ; L^{2}(A)\right) \cap L^{2}\left(0, \tau ; H^{1}(A)\right), \\
T_{B} \in C\left([0, \tau] ; L^{2}(B)\right) \cap L^{2}\left(0, \tau ; H^{1}(B)\right) .
\end{array}\right.
$$

In that case the functions $T_{A}$ and $T_{B}$ have traces on $\partial B$ denoted $\left.T_{A}\right|_{\partial B}$ and $\left.T_{B}\right|_{\partial B}$ belonging to $L^{2}\left(0, \tau ; H^{1 / 2}(\partial B)\right)$.

Moreover, if $T_{A}$ and $T_{B}$ satisfy (1.2), the vector fields

$$
\left(\rho_{A} C_{A} T_{A},-\kappa_{A} \nabla_{x} T_{A}\right) \text { and }\left(\rho_{B} C_{B} T_{B},-\kappa_{B} \nabla_{x} T_{B}\right)
$$

are divergence free in $(0, \tau) \times A$ and $(0, \tau) \times \stackrel{\circ}{B}$, respectively. By statement (a) in Lemma A.3, both sides of the second equality in (1.3) are well defined elements of $H_{00}^{1 / 2}((0, \tau) \times \partial B)^{\prime}$. (We recall that $H_{00}^{1 / 2}((0, \tau) \times \partial B)$ is the Lions-Magenes subspace of functions in $H^{1 / 2}((0, \tau) \times \partial B)$ whose extension by 0 to $\mathbf{R} \times \partial B$ defines an element of $H^{1 / 2}(\mathbf{R} \times \partial B)$; the notation $H_{00}^{1 / 2}((0, \tau) \times \partial B)^{\prime}$ designates the dual of that space. $)$

Lemma 1.1. Assume that $T_{A}$ and $T_{B}$ satisfy assumptions (1.7). Let $\rho, C, \kappa$ and $T$ be defined as in (1.4), (1.5) and (1.6). Then

$$
T \in C\left([0, \tau] ; L^{2}(\Omega)\right) \cap L^{2}\left(0, \tau ; H^{1}(\Omega)\right)
$$

and

$$
\rho(x) C(x) \partial_{t} T(t, x)=\operatorname{div}_{x}\left(\kappa(x) \nabla_{x} T(t, x)\right) \quad x \in \Omega, t>0
$$

holds in the sense of distributions in $(0, \tau) \times \Omega$ if and only if both (1.2) and (1.3) hold in the sense of distributions.

Proof. Under the assumption (1.7), the function $T$ defined by (1.6) belongs to the space $L^{2}\left((0, \tau) ; H^{1}(\Omega)\right)$ if and only if the boundary traces of $T_{A}$ and $T_{B}$ coincide, i.e.

$$
\left.T_{A}(t, \cdot)\right|_{\partial B}=\left.T_{B}(t, \cdot)\right|_{\partial B} \quad \text { for a.e. } t \in[0, \tau] .
$$

If (1.8) holds in the sense of distributions on $(0, \tau) \times \Omega$, then $(1.2)$ hold in the sense of distributions on $(0, \tau) \times A$ and $(0, \tau) \times B$, respectively. 
For $\phi \in C_{c}^{\infty}(\Omega)$, one has

$$
\begin{array}{r}
\frac{\mathrm{d}}{\mathrm{d} t} \int_{\Omega} \rho(x) C(x) T(t, x) \phi(x) \mathrm{d} x+\int_{\Omega} \kappa(x) \nabla_{x} T(t, x) \cdot \nabla \phi(x) \mathrm{d} x \\
=\frac{\mathrm{d}}{\mathrm{d} t} \int_{A} \rho_{A}(x) C_{A}(x) T_{A}(t, x) \phi(x) \mathrm{d} x+\int_{A} \kappa_{A}(x) \nabla_{x} T_{A}(t, x) \cdot \nabla \phi(x) \mathrm{d} x \\
+\frac{\mathrm{d}}{\mathrm{d} t} \int_{B} \rho_{B}(x) C_{B}(x) T_{B}(t, x) \phi(x) \mathrm{d} x+\int_{B} \kappa_{B}(x) \nabla_{x} T_{B}(t, x) \cdot \nabla \phi(x) \mathrm{d} x \\
=-\left\langle\kappa_{A} \frac{\partial T_{A}}{\partial n}, \phi\right\rangle_{H^{-1 / 2}(\partial B), H^{1 / 2}(\partial B)}+\left\langle\kappa_{B} \frac{\partial T_{B}}{\partial n}, \phi\right\rangle_{H^{-1 / 2}(\partial B), H^{1 / 2}(\partial B)}
\end{array}
$$

provided that $T_{A}$ and $T_{B}$ satisfy (1.2), by statement (b) in Lemma A.3.

Thus, if $T$ satisfies (1.8) in the sense of distributions on $(0, \tau) \times \Omega$, then $T_{A}$ and $T_{B}$ satisfy $(1.2)$ on $(0, \tau) \times A$ and $(0, \tau) \times \stackrel{\circ}{B}$, respectively. Therefore the identity above holds with left hand side equal to 0 in the sense of distributions on $(0, \tau)$, so that

$$
\left\langle\kappa_{A} \frac{\partial T_{A}}{\partial n}-\kappa_{B} \frac{\partial T_{B}}{\partial n}, \phi\right\rangle_{H^{-1 / 2}(\partial B), H^{1 / 2}(\partial B)}=0 \quad \text { in } \mathcal{D}^{\prime}((0, \tau)) .
$$

This implies in turn the second equality in (1.3).

Conversely, if $T_{A}$ and $T_{B}$ satisfy (1.2), the above identity holds with right hand side equal to 0 by the second equality in (1.3). Therefore

$$
\frac{\mathrm{d}}{\mathrm{d} t} \int_{\Omega} \rho(x) C(x) T(t, x) \phi(x) \mathrm{d} x+\int_{\Omega} \kappa(x) \nabla_{x} T(t, x) \cdot \nabla \phi(x) \mathrm{d} x=0
$$

for all $\phi \in C_{c}^{\infty}(\Omega)$, which implies that (1.8) holds in the sense of distributions on $(0, \tau) \times \Omega$ by a classical density argument.

Therefore, we start from the heat equation (1.8) with $\rho, C, \kappa$ as in (1.4), (1.5) and we assume that there is no heat flux across $\partial \Omega$, in other words that $T$ satisfies the Neumann boundary condition

$$
\frac{\partial T}{\partial n}(t, x)=0, \quad x \in \partial \Omega, t>0 .
$$

\subsection{The model with infinite conductivity}

In this section we assume that $B$ has $N$ connected components denoted $B_{i}$ for $i=1, \ldots, N$.

Our first task is to derive the governing equation for the temperature field $T$ in $\Omega$ when the material $\mathcal{B}$ filling $B$ has infinite heat conductivity. In that case the temperature $T$ instantaneously reaches equilibrium in each connected component $B_{i}$ of $B$, so that

$$
T(t, x)=T_{i}(t), \quad x \in B_{i}, t>0,
$$

for each $i=1, \ldots, N$. Therefore, the unknown for the problem with infinite conductivity is $\left(T_{A}(t, x), T_{1}(t), \ldots, T_{N}(t)\right)$, where

$$
\begin{cases}\rho_{A}(x) C_{A}(x) \partial_{t} T_{A}(t, x)=\operatorname{div}_{x}\left(\kappa_{A}(x) \nabla_{x} T_{A}(t, x)\right), & x \in A, t>0, \\ \frac{\partial T_{A}}{\partial n}(t, x)=0, & x \in \partial \Omega, t>0 \\ T_{A}(t, x)=T_{i}(t), & x \in \partial B_{i}, t>0\end{cases}
$$


This is obviously not enough to determine the evolution of $T_{A}$ and of $T_{i}$ for all $i=1, \ldots, N$.

For finite $\kappa_{B}$, the vector field

$$
(t, x) \mapsto\left(\rho_{B}(x) C_{B}(x) T_{B}(t, x),-\kappa_{B}(x) \nabla_{x} T_{B}(t, x)\right)
$$

is divergence free in $(0, \tau) \times B_{i}$ for each $i=1, \ldots, N$. By statement (b) in Lemma A.3 and the second equality in $(1.3)$

$$
\begin{aligned}
\frac{\mathrm{d}}{\mathrm{d} t} \int_{B_{i}} \rho_{B}(x) C_{B}(x) T_{B}(t, x) \mathrm{d} x & =\left\langle\kappa_{B}(x) \frac{\partial T_{B}}{\partial n}(t, \cdot), 1\right\rangle_{H^{-1 / 2}\left(\partial B_{i}\right), H^{1 / 2}\left(\partial B_{i}\right)} \\
& =\left\langle\kappa_{A}(x) \frac{\partial T_{A}}{\partial n}(t, \cdot), 1\right\rangle_{H^{-1 / 2}\left(\partial B_{i}\right), H^{1 / 2}\left(\partial B_{i}\right)} .
\end{aligned}
$$

Letting $\kappa_{B} \rightarrow \infty$ and abusing the integral notation to designate the last duality bracket above, one uses (1.10) to conclude that

$$
\dot{T}_{i}(t)=\frac{1}{\beta_{i}} \int_{\partial B_{i}} \kappa_{A}(x) \frac{\partial T_{A}}{\partial n}(t, x) \mathrm{d} S(x)
$$

where

$$
\beta_{i}:=\int_{B_{i}} \rho_{B}(x) C_{B}(x) \mathrm{d} x .
$$

The argument above suggests that the governing equations for the infinite conductivity problem with unknowns $\left(T_{A}(t, x), T_{1}(t), \ldots, T_{N}(t)\right)$ is the system consisting of (1.11) with (1.12) for $i=1, \ldots, N$. This model is analogous to the system obtained by Bal in the context of the diffusion approximation for the transport equation with nondiffusive inclusions (see Eqs. (3.2)-(3.4) in [1]).

\section{MAin RESUlts}

\subsection{Existence and uniqueness theory for the heat equation with discontinuous coefficients}

Since our starting point is (1.2) with interface condition (1.3), or equivalently the heat equation (1.8) with discontinuous coefficients (see Lem. 1.1), we first recall the existence and uniqueness theory for (1.8) with Neumann boundary condition (1.9). Except for the possibly non smooth factor $\rho(x) C(x)$, this is a classical result. This factor can be handled with appropriate weighted Sobolev spaces; for the sake of being complete, we sketch the (elementary) argument below.

Proposition 2.1. Let $\kappa \equiv \kappa(x), \rho \equiv \rho(x)$ and $C \equiv C(x)$ be measurable functions on $\Omega$ satisfying

$$
\kappa_{m} \leq \kappa(x) \leq \kappa_{M}, \quad \rho_{m} \leq \rho(x) \leq \rho_{M}, \quad C_{m} \leq C(x) \leq C_{M}
$$

for a.e. $x \in \Omega$, where $\kappa_{m}, \kappa_{M}, \rho_{m}, \rho_{M}, C_{m}, C_{M}>0$, and let $T^{i n} \in L^{2}(\Omega)$. There exists a unique

$$
T \in C_{b}\left([0,+\infty) ; L^{2}(\Omega)\right) \cap L^{2}\left(0, \tau ; H^{1}(\Omega)\right)
$$

for each $\tau>0$ that is a weak solution to the problem

$$
\begin{cases}\rho(x) C(x) \partial_{t} T(t, x)=\operatorname{div}_{x}\left(\kappa(x) \nabla_{x} T(t, x)\right), & x \in \Omega, t>0 \\ \frac{\partial T}{\partial n}(t, x)=0, & x \in \partial \Omega, t>0 \\ T(0, x)=T^{i n}(x), & x \in \Omega .\end{cases}
$$

This solution satisfies

$$
\rho C \partial_{t} T \in L^{2}\left(0, \tau ; H^{1}(\Omega)^{\prime}\right),
$$


for each $\tau>0$, together with the "energy" identity

$$
\frac{1}{2} \int_{\Omega} \rho(x) C(x) T(t, x)^{2} \mathrm{~d} x+\int_{0}^{t} \int_{\Omega} \kappa(x)\left|\nabla_{x} T(t, x)\right|^{2} \mathrm{~d} x \mathrm{~d} t=\frac{1}{2} \int_{\Omega} \rho(x) C(x) T^{i n}(x)^{2} \mathrm{~d} x
$$

for each $t>0$.

We recall the weak formulation of (2.1): for each $w \in H^{1}(\Omega)$

$$
\left\langle\rho C \partial_{t} T(t, \cdot), w\right\rangle_{H^{1}(\Omega)^{\prime}, H^{1}(\Omega)}+\int_{\Omega} \kappa(x) \nabla_{x} T(t, x) \cdot \nabla w(x) \mathrm{d} x=0 \text { for } \text { a.e. } t \geq 0 .
$$

The Neumann condition in $(2.1)$ is contained in the choice of $L^{2}\left([0,+\infty) ; H^{1}(\Omega)\right)$ as the set of test functions in the weak formulation above, while there is no difficulty with initial condition since $T \in C\left([0,+\infty) ; L^{2}(\Omega)\right)$.

\subsection{The infinite conductivity limit}

\subsubsection{Variational formulation of the infinite conductivity problem}

Assume as in Section 1.3 that $B$ has $N$ connected components denoted $B_{i}$ for $i=1, \ldots, N$. The heat diffusion problem with infinite heat conductivity in $B$ is:

$$
\begin{cases}\rho_{A} C_{A} \partial_{t} T(t, x)=\operatorname{div}_{x}\left(\kappa_{A} \nabla_{x} T(t, x)\right), & x \in A, t>0, \\ \frac{\partial T}{\partial n}(t, x)=0, & x \in \partial \Omega, t>0, \\ T(t, x)=T_{i}(t), & x \in \partial B_{i}, t>0,1 \leq i \leq N, \\ \beta_{i} \dot{T}_{i}(t)=\int_{\partial B_{i}} \kappa_{A} \frac{\partial T}{\partial n}(t, x) \mathrm{d} S(x), & t>0,1 \leq i \leq N, \\ T(0, x)=T^{i n}(x), & x \in \Omega,\end{cases}
$$

where we recall that the outward unit normal field $n$ on $\partial B$ is directed toward $A$. Its variational formulation is as follows.

Let $\mathcal{H}_{N}$ be the closed subspace of $L^{2}(\Omega)$ defined as

$$
\mathcal{H}_{N}:=\left\{u \in L^{2}(\Omega) \text { s.t. } u(x)=\frac{1}{\left|B_{i}\right|} \int_{B_{i}} u(y) \mathrm{d} y \text { for a.e. } x \in B_{i}, i=1, \ldots, N\right\}
$$

and equipped with the inner product

$$
(u \mid v)_{\mathcal{H}_{N}}=\int_{\Omega} u(x) v(x) \rho(x) C(x) \mathrm{d} x .
$$

Define

$$
\mathcal{V}_{N}:=\mathcal{H}_{N} \cap H^{1}(\Omega)
$$

with the inner product

$$
(u \mid v)_{\mathcal{V}_{N}}=(u \mid v)_{\mathcal{H}_{N}}+\int_{A} \nabla u(x) \cdot \nabla v(x) \rho_{A}(x) C_{A}(x) \mathrm{d} x .
$$

Obviously $\mathcal{V}_{N}$ is a separable Hilbert space, the inclusion $\mathcal{V}_{N} \subset \mathcal{H}_{N}$ is continuous and $\mathcal{V}_{N}$ is a dense subspace of $\mathcal{H}_{N}$. Besides, the map $\mathcal{H}_{N} \ni u \mapsto L_{u} \in \mathcal{V}_{N}^{\prime}$, where $L_{u}$ is the linear functional $v \mapsto(u \mid v)_{\mathcal{H}_{N}}$, identifies $\mathcal{H}_{N}$ with a dense subspace of $\mathcal{V}_{N}^{\prime}$. 
The variational formulation of the infinite conductivity problem is as follows: a weak solution to (2.2) is a function

$$
T \in C\left([0, \tau] ; \mathcal{H}_{N}\right) \cap L^{2}\left(0, \tau ; \mathcal{V}_{N}\right) \text { such that } \rho C \partial_{t} T \in L^{2}\left(0, \tau ; \mathcal{V}_{N}^{\prime}\right)
$$

satisfying the initial condition and

$$
\left\{\begin{array}{l}
\partial_{t}(T(t, \cdot) \mid w)_{\mathcal{H}_{N}}+\int_{A} \kappa_{A}(x) \nabla_{x} T(t, x) \cdot \nabla w(x) \mathrm{d} x=0 \text { for a.e. } t \in[0, \tau] \\
\text { for each test function } w \in \mathcal{V}_{N} .
\end{array}\right.
$$

This variational formulation is justified by the following observation.

Proposition 2.2. Let $T$ satisfy (2.3) and the initial condition in (2.2).

If $T$ satisfies the variational condition (2.4), then

$$
\rho_{A} C_{A} \partial_{t} T=\operatorname{div}_{x}\left(\kappa_{A} \nabla_{x} T\right) \quad \text { in } \mathcal{D}^{\prime}((0, \tau) \times A)
$$

and

while

$$
\left.\kappa_{A} \frac{\partial T}{\partial n}\right|_{(0, \tau) \times \partial \Omega}=0 \quad \text { in } H_{00}^{1 / 2}((0, \tau) \times \partial \Omega)^{\prime}
$$

$$
\beta_{i} \dot{T}_{i}=\left\langle\left.\kappa_{A} \frac{\partial T}{\partial n}\right|_{\partial B_{i}}, 1\right\rangle_{H^{-1 / 2}\left(\partial B_{i}\right), H^{1 / 2}\left(\partial B_{i}\right)} \text { in } H^{-1}((0, \tau))
$$

for each $i=1, \ldots, N$, where

$$
T_{i}(t):=\frac{1}{\left|B_{i}\right|} \int_{B_{i}} T(t, x) \mathrm{d} x .
$$

Conversely, if $T$ satisfies both (2.5), (2.6) and (2.7), it must satisfy the variational formulation (2.4).

The existence and uniqueness of a weak solution to the infinite heat conductivity problem is given in the next proposition.

Proposition 2.3. Assume that $\kappa_{A}$ is a measurable function defined a.e. on A satisfying

$$
\kappa_{m} \leq \kappa_{A}(x) \leq \kappa_{M}, \quad \text { for a.e. } x \in A,
$$

where $\kappa_{m}$ and $\kappa_{M}$ are positive numbers, while $\rho$ and $C$ satisfy the same assumptions as in Proposition 2.1. Then for each $T^{\text {in }} \in \mathcal{H}_{N}$, there exists a unique weak solution $T$ to $(2.2)$ defined for all $t \in[0,+\infty)$. This solution satisfies

$$
\rho C \partial_{t} T \in L^{2}\left(0, \tau ; \mathcal{V}_{N}^{\prime}\right)
$$

for all $\tau>0$, together with the "energy" identity

$$
\begin{aligned}
\frac{1}{2} \int_{A} \rho_{A}(x) C_{A}(x) T(t, x)^{2} \mathrm{~d} x & +\frac{1}{2} \sum_{i=1}^{N} \beta_{i} T_{i}(t)^{2}+\int_{0}^{t} \int_{A} \kappa_{A}(x)\left|\nabla_{x} T(t, x)\right|^{2} \mathrm{~d} x \mathrm{~d} t \\
& =\frac{1}{2} \int_{A} \rho_{A}(x) C_{A}(x) T^{i n}(x)^{2} \mathrm{~d} x+\frac{1}{2} \sum_{i=1}^{N} \beta_{i}\left|T_{i}^{i n}\right|^{2}
\end{aligned}
$$

for each $t>0$, where

$$
T_{i}(t):=\frac{1}{\left|B_{i}\right|} \int_{B_{i}} T(t, y) \mathrm{d} y \quad \text { and } \quad T_{i}^{i n}:=\frac{1}{\left|B_{i}\right|} \int_{B_{i}} T^{i n}(y) \mathrm{d} y .
$$


Notice that this existence and uniqueness result assumes that the initial temperature field $T^{i n}$ is a constant in each connected component of $B$. This assumption is implied by the requirement that $T^{i n} \in \mathcal{H}_{N}$. While this restriction may seem questionable, it is very natural from the mathematical viewpoint. For general initial temperature fields $T^{i n}$, the solution to (2.2) would include an initial layer corresponding with the relaxation to thermal equilibrium in each connected component of $B$. Such initial layers involve fast variations of the temperature field that are incompatible with the condition $\rho C \partial_{t} T \in L^{2}\left(0, \tau ; \mathcal{V}_{N}^{\prime}\right)$ in the infinite conductivity limit.

\subsubsection{Convergence to the infinite conductivity model}

For each $\eta>0$, let $\kappa_{\eta}$ be defined as follows:

$$
\kappa_{\eta}(x):= \begin{cases}\kappa_{A}(x) & x \in A \\ \kappa_{B}(x) / \eta & x \in B\end{cases}
$$

where $\kappa_{A}$ and $\kappa_{B}$ are measurable functions on $A$ and $B$, respectively, satisfying

$$
\kappa_{m} \leq \kappa_{A}(x) \leq \kappa_{M} \text { and } \kappa_{m} \leq \kappa_{B}(y) \leq \kappa_{M}, \quad \text { for a.e. } x \in A \text { and } y \in B,
$$

$\kappa_{M}$ and $\kappa_{m}$ being two positive constants.

Theorem 2.4. Assume that $\rho$ and $C$ satisfy the same assumptions as in Proposition 2.1, while $\kappa_{A}$ and $\kappa_{B}$ satisfy (2.10). Let $T^{i n} \in \mathcal{H}_{N}$. For each $\eta>0$, let $T_{\eta} \in C_{b}\left([0,+\infty) ; L^{2}(\Omega)\right) \cap L^{2}\left(0, \tau ; H^{1}(\Omega)\right)$ for all $\tau>0$ be the weak solution to (2.1) with heat conductivity $\kappa_{\eta}$ defined as in (2.9) and initial data $T^{i n}$. Then

$$
T_{\eta} \rightarrow T \text { in } L^{2}\left(0, \tau ; H^{1}(\Omega)\right)
$$

as $\eta \rightarrow 0$ for all $\tau>0$, where $T \in C_{b}\left([0,+\infty) ; \mathcal{H}_{N}\right) \cap L^{2}\left(0, \tau ; \mathcal{V}_{N}\right)$ for all $\tau>0$ is the weak solution to the infinite conductivity problem (2.2).

\subsection{The homogenized system}

Let $\sigma, \sigma^{\prime}>0$ and let $\varrho \in C_{b}(\bar{\Omega})$ be a probability density on $\bar{\Omega}$ such that $1 / \varrho$ is bounded on $\bar{\Omega}$. Let $T^{\text {in }}, \vartheta^{\text {in }} \in$ $L^{2}(\Omega)$. Consider the system

$$
\begin{cases}\left(\partial_{t}-\sigma \Delta_{x}\right) T(t, x)+4 \pi \sigma(\varrho(x) T(t, x)-\vartheta(t, x))=0, & x \in \Omega, t>0, \\ \partial_{t} \vartheta(t, x)+4 \pi \sigma^{\prime}(\vartheta(t, x)-\varrho(x) T(t, x))=0, & x \in \Omega, t>0, \\ \frac{\partial T}{\partial n}(t, x)=0, & x \in \partial \Omega, t>0, \\ T(0, x)=T^{i n}(x), \quad \vartheta(0, x)=\vartheta^{i n}(x), & x \in \Omega .\end{cases}
$$

A weak solution to $(2.11)$ is a pair $(T, \vartheta)$ such that

$$
T \in L^{\infty}\left([0,+\infty) ; L^{2}(\Omega)\right) \cap L^{2}\left(0, \tau ; H^{1}(\Omega)\right) \quad \text { and } \vartheta \in L^{\infty}\left([0,+\infty) ; L^{2}(\Omega)\right),
$$

for all $\tau>0$, and

$$
\begin{aligned}
\frac{\mathrm{d}}{\mathrm{d} t} \int_{\Omega} T(t, x) \phi(x) \mathrm{d} x & +\sigma \int_{\Omega} \nabla_{x} T(t, x) \cdot \nabla \phi(x) \mathrm{d} x \\
& +4 \pi \sigma \int_{\Omega}(\varrho(x) T(t, x)-\vartheta(t, x)) \phi(x) \mathrm{d} x=0, \\
\frac{\mathrm{d}}{\mathrm{d} t} \int_{\Omega} \vartheta(t, x) \psi(x) \mathrm{d} x & +4 \pi \sigma^{\prime} \int_{\Omega}(\vartheta(t, x)-\varrho(x) T(t, x)) \psi(x) \mathrm{d} x=0,
\end{aligned}
$$


in the sense of distributions on $(0,+\infty)$ for each $\phi \in H^{1}(\Omega)$ and $\psi \in L^{2}(\Omega)$, together with the initial condition. Observe that the identities above imply that

$$
\frac{\mathrm{d}}{\mathrm{d} t} \int_{\Omega} T(t, x) \phi(x) \mathrm{d} x \quad \text { and } \frac{\mathrm{d}}{\mathrm{d} t} \int_{\Omega} \vartheta(t, x) \psi(x) \mathrm{d} x \in L^{2}([0, \tau])
$$

for each $\tau>0$, so that the functions

$$
t \mapsto \int_{\Omega} T(t, x) \phi(x) \mathrm{d} x \quad \text { and } t \mapsto \int_{\Omega} \vartheta(t, x) \psi(x) \mathrm{d} x
$$

are continuous on $[0,+\infty)$. Therefore the initial condition, interpreted as

$$
\int_{\Omega} T(0, x) \phi(x) \mathrm{d} x=\int_{\Omega} T^{i n}(x) \phi(x) \mathrm{d} x, \quad \int_{\Omega} \vartheta(0, x) \psi(x) \mathrm{d} x=\int_{\Omega} \vartheta^{i n}(x) \psi(x) \mathrm{d} x
$$

for all $\phi \in H^{1}(\Omega)$ and all $\psi \in L^{2}(\Omega)$, makes perfect sense.

In the next proposition, we state the basic results concerning the existence and uniqueness of a weak solution to the initial-boundary value problem for the homogenized system. In fact, one can say more about the continuity in time of $(T, \vartheta)$, as explained below.

Proposition 2.5. Under the assumptions above, any weak solution to (2.11) satisfies

$$
\partial_{t} T \in L^{2}\left(0, \tau ; H^{1}(\Omega)^{\prime}\right) \quad \text { and } \partial_{t} \vartheta \in L^{2}\left(0, \tau ; L^{2}(\Omega)\right),
$$

and (up to modification on some negligible t-set)

$$
T, \vartheta \in C_{b}\left([0,+\infty) ; L^{2}(\Omega)\right) .
$$

Moreover, there exists a unique weak solution to the system (2.11). It is a solution to the partial differential equations

$$
\left\{\begin{array}{l}
\partial_{t} T-\sigma \Delta_{x} T+4 \pi \sigma(\varrho T-\vartheta)=0 \\
\partial_{t} \vartheta+4 \pi \sigma^{\prime}(\vartheta-\varrho T)=0
\end{array}\right.
$$

in the sense of distributions on $(0,+\infty) \times \Omega$, and satisfies the Neumann condition

$$
\left.\frac{\partial T}{\partial n}\right|_{(0, \tau) \times \partial \Omega}=0
$$

in $H_{00}^{1 / 2}((0, \tau) \times \partial \Omega)^{\prime}$ for each $\tau>0$.

In fact, the existence of the solution to (2.11) follows from Theorem 2.6

\subsection{The homogenization limit}

Henceforth we assume that the material $\mathcal{B}$ occupies $N$ identical spherical inclusions with radius $\epsilon$ :

$$
B_{\epsilon}=\bigcup_{i=1}^{N} B_{i} \quad \text { where } B_{i}:=\overline{B\left(x_{i}, \epsilon\right)}, \quad i=1, \ldots, N
$$

and henceforth denote

$$
A_{\epsilon}=\Omega \backslash B_{\epsilon}
$$


The number of inclusions $N$ is assumed to scale as

$$
N=1 / \epsilon
$$

The inclusion centers $x_{i}$ are distributed so that their empirical distribution satisfies

$$
\frac{1}{N} \sum_{i=1}^{N} \delta_{x_{i}} \rightarrow \varrho \mathscr{L}^{3}
$$

in the weak topology of probability measures, where $\mathscr{L}^{3}$ designates the 3 -dimensional Lebesgue measure and

$$
\varrho \text { and } 1 / \varrho \in C_{b}(\bar{\Omega}), \quad \int_{\Omega} \varrho(x) \mathrm{d} x=1 .
$$

Finally, we denote

$$
r_{\epsilon}=\epsilon^{1 / 3}
$$

and assume that the inclusion centers are chosen so that

$$
\left|x_{i}-x_{j}\right|>2 r_{\epsilon} \quad \text { for all } i \neq j \text { with } i, j=1, \ldots, N .
$$

For simplicity we assume that $\rho_{A}, C_{A}$ and $\kappa_{A}$ are constants, and define

$$
\sigma=\kappa_{A} / \rho_{A} C_{A} .
$$

We further assume that $\rho_{B}$ and $C_{B}$ are scaled with $\epsilon$ so that $\rho_{B} C_{B} \sim$ Const. $/ \epsilon^{2}$, and introduce the constant

$$
\sigma^{\prime}=3 \kappa_{A} / 4 \pi \rho_{B} C_{B} \epsilon^{2} .
$$

The scaled infinite heat conductivity problem takes the form

$$
\begin{cases}\partial_{t} T_{\epsilon}(t, x)=\sigma \Delta_{x} T_{\epsilon}(t, x), & x \in A_{\epsilon}, t>0, \\ \frac{\partial T_{\epsilon}}{\partial n}(t, x)=0, & x \in \partial \Omega, t>0, \\ T_{\epsilon}(t, x)=T_{i, \epsilon}(t), & x \in \partial B\left(x_{i}, \epsilon\right), t>0,1 \leq i \leq N, \\ \dot{T}_{i, \epsilon}(t)=\frac{\sigma^{\prime}}{\epsilon} \int_{\partial B\left(x_{i}, \epsilon\right)} \frac{\partial T_{\epsilon}}{\partial n}(t, x) \mathrm{d} S(x), & t>0,1 \leq i \leq N, \\ T_{\epsilon}(0, x)=T_{\epsilon}^{i n}(x), & x \in \Omega .\end{cases}
$$

The initial data $T_{\epsilon}^{i n} \in \mathcal{H}_{N}$, so that $T_{\epsilon}^{i n}$ is a.e. a constant in $B\left(x_{i}, \epsilon\right)$ :

$$
T_{i, \epsilon}^{i n}:=\frac{3}{4 \pi \epsilon^{3}} \int_{B\left(x_{i}, \epsilon\right)} T^{i n}(x) \mathrm{d} x .
$$

Then

$$
\begin{aligned}
\left|T_{\epsilon}^{i n}\right|_{\mathcal{H}_{N}}^{2} & =\rho_{A} C_{A} \int_{A_{\epsilon}} T_{\epsilon}^{i n}(x)^{2} \mathrm{~d} x+\sum_{i=1}^{N} \frac{4 \pi}{3} \rho_{B} C_{B} \epsilon^{3}\left|T_{i, \epsilon}^{i n}\right|^{2} \\
& =\rho_{A} C_{A}\left(\int_{A_{\epsilon}} T_{\epsilon}^{i n}(x)^{2} \mathrm{~d} x+\frac{\sigma}{\sigma^{\prime}} \epsilon \sum_{i=1}^{N}\left|T_{i, \epsilon}^{i n}\right|^{2}\right) .
\end{aligned}
$$

We shall henceforth assume that the initial data satisfies

$$
\left|T_{\epsilon}^{i n}\right|_{\mathcal{H}_{N}}^{2}=O(1)
$$


i.e. that there exists a positive constant, taken equal to $C^{\text {in }}$ for notational simplicity, such that

$$
\int_{A_{\epsilon}} T_{\epsilon}^{i n}(x)^{2} \mathrm{~d} x+\frac{\sigma}{\sigma^{\prime}} \epsilon \sum_{i=1}^{N}\left|T_{i, \epsilon}^{i n}\right|^{2} \leq C^{i n} \quad \text { for all } \epsilon>0 .
$$

Theorem 2.6. Assume that (2.14) holds, that the distribution of inclusion centers satisfies (2.15) and (8.1), that the volumetric heat capacity of the material in the inclusions scales as prescribed in (2.20), and that the initial data $T_{\epsilon}^{i n} \in \mathcal{H}_{N}$ satisfy the bound (2.23). Assume further that

$$
T_{\epsilon}^{i n} \rightarrow T^{\text {in }} \quad \text { in } L^{2}(\Omega) \text { weak as } \epsilon \rightarrow 0
$$

while 4

$$
\frac{1}{N} \sum_{i=1}^{N} T_{i, \epsilon}^{i n} \delta_{x_{i}} \rightarrow \vartheta^{\text {in }} \text { in } \mathcal{M}_{b}(\Omega) \text { weak-* as } \epsilon \rightarrow 0 .
$$

Let $T_{\epsilon} \in C\left([0,+\infty) ; \mathcal{H}_{N}\right) \cap L^{2}\left(0, \tau, \mathcal{V}_{N}\right)$ for all $\tau>0$ be the weak solution to the scaled infinite heat conductivity problem (2.21). Then, in the limit as $\epsilon \rightarrow 0$,

$$
T_{\epsilon} \rightarrow T\left\{\begin{array}{l}
\text { in } L^{2}\left(0, \tau ; H^{1}(\Omega)\right) \text { weak for all } \tau>0 \\
\text { and in } L^{\infty}\left([0,+\infty) ; L^{2}(\Omega)\right) \text { weak-* }
\end{array}\right.
$$

and

$$
\vartheta_{\epsilon}:=\frac{1}{N} \sum_{i=1}^{N} T_{i, \epsilon} \delta_{x_{i}} \rightarrow \vartheta \text { in } L^{\infty}\left([0,+\infty) ; \mathcal{M}_{b}(\Omega)\right) \text { weak-* }
$$

where

$$
T_{i, \epsilon}:=\frac{3}{4 \pi \epsilon^{3}} \int_{B\left(x_{i}, \epsilon\right)} T_{\epsilon}(t, x) \mathrm{d} x .
$$

Besides

$$
T \in C_{b}\left([0,+\infty) ; L^{2}(\Omega)\right) \times L^{2}\left(0, \tau ; H^{1}(\Omega)\right) \text { for each } \tau>0
$$

while

$$
\vartheta \in C_{b}\left([0,+\infty) ; L^{2}(\Omega)\right) .
$$

Finally, the pair $(T, \vartheta)$ is the unique weak solution to the homogenized system (2.11) with initial condition

$$
\left.T\right|_{t=0}=T^{i n},\left.\quad \vartheta\right|_{t=0}=\vartheta^{i n} .
$$

\section{Proofs of Propositions 2.1, 2.2 And 2.3}

Proof of Proposition 2.1. Consider the Hilbert spaces $\mathcal{H}=L^{2}(\Omega)$ and $\mathcal{V}=H^{1}(\Omega)$ equipped with the inner products

$$
\begin{aligned}
(u \mid v)_{\mathcal{H}} & :=\int_{\Omega} u(x) v(x) \rho(x) C(x) \mathrm{d} x, \\
(u \mid v)_{\mathcal{V}} & :=\int_{\Omega}(u(x) v(x)+\nabla u(x) \cdot \nabla v(x)) \rho(x) C(x) \mathrm{d} x .
\end{aligned}
$$

Let $a$ be the bilinear form defined on $\mathcal{V} \times \mathcal{V}$ by

$$
a(u, v)=\int_{\Omega} \kappa(x) \nabla_{x} u(x) \cdot \nabla_{x} v(x) \mathrm{d} x
$$

\footnotetext{
${ }^{4}$ The notation $\mathcal{M}_{b}(\Omega)$ designates the set of bounded (signed) Radon measures on $\Omega$.
} 
observe that

$$
|a(u, v)| \leq \frac{\kappa_{M}}{\rho_{m} C_{m}}(u \mid u)_{\mathcal{V}}^{1 / 2}(v \mid v)_{\mathcal{V}}^{1 / 2}
$$

while

$$
a(u, u) \geq \frac{\kappa_{m}}{\rho_{M} C_{M}}\left((u \mid u)_{\mathcal{V}}-(u \mid u)_{\mathcal{H}}\right) .
$$

By Theorem X.9 in [7] (see also Chap. 3, Sect. 4 in [12], Thm. 7.2.1 in [16] or Thm. 26.1 in [17]), there exists a unique $T \in L^{2}(0, \tau ; \mathcal{V}) \cap C_{b}([0, \tau] ; \mathcal{H})$ such that $\rho C \partial_{t} T \in L^{2}\left(0, \tau ; \mathcal{V}^{\prime}\right)$ for each $\tau>0$ such that the linear functional

$$
L(t): w \mapsto \partial_{t}(T(t, \cdot) \mid w)_{\mathcal{H}}+a(T(t, \cdot), w)=\left\langle\rho C \partial_{t} T(t, \cdot), w\right\rangle_{\mathcal{V}^{\prime}, \mathcal{V}}+a(T(t, \cdot), w)
$$

satisfies

$$
\langle L(t), w\rangle_{\mathcal{V}^{\prime}, \mathcal{V}}=0 \text { for a.e. } t \in[0,+\infty)
$$

for all $w \in \mathcal{V}$. Equivalently, $T$ is the unique weak solution to $(2.1)$.

By Lemma A.2, this linear functional satisfies $L(t)=0$ for a.e. $t \in[0,+\infty)$. In particular

$$
0=\langle L(s), T(s, \cdot)\rangle_{\mathcal{V}^{\prime}, \mathcal{V}}=\left\langle\rho C \partial_{t} T(s, \cdot), T(s, \cdot)\right\rangle_{\mathcal{V}^{\prime}, \mathcal{V}}+a(T(s, \cdot), T(s, \cdot))
$$

for a.e. $s \in[0,+\infty)$. Integrating in $s \in[0, t]$ and applying statement (b) of Lemma A.1 give the "energy identity".

Proof of Proposition 2.2. Specializing (2.4) to the case where $w \in C_{c}^{\infty}(A)$ implies (2.5). In particular, the vector field

$$
(0, \tau) \times A \ni(t, x) \mapsto\left(\rho_{A}(x) C_{A}(x) T(t, x),-\kappa_{A}(x) \nabla_{x} T(t, x)\right)
$$

is divergence free in $(0, \tau) \times A$. Applying statement (b) in Lemma A.3 shows that, for each $w \in \mathcal{V}_{N}$, one has

$$
\begin{aligned}
0= & \frac{\mathrm{d}}{\mathrm{d} t} \int_{\Omega} \rho(x) C(x) T(t, x) w(x) \mathrm{d} x+\int_{A} \kappa_{A}(x) \nabla_{x} T(t, x) \cdot \nabla w(x) \mathrm{d} x \\
= & \frac{\mathrm{d}}{\mathrm{d} t} \int_{A} \rho_{A}(x) C_{A}(x) T(t, x) w(x) \mathrm{d} x+\sum_{i=1}^{N} \beta_{i} w_{i} \dot{T}_{i}(t)+\int_{A} \kappa_{A}(x) \nabla_{x} T(t, x) \cdot \nabla w(x) \mathrm{d} x \\
= & \sum_{i=1}^{N} w_{i}\left(\beta_{i} \dot{T}_{i}(t)-\left\langle\left.\kappa_{A} \frac{\partial T}{\partial n}(t, \cdot)\right|_{\partial B_{i}}, 1\right\rangle_{H^{-1 / 2}\left(\partial B_{i}\right), H^{1 / 2}\left(\partial B_{i}\right)}\right) \\
& +\left\langle\left.\kappa_{A} \frac{\partial T}{\partial n}(t, \cdot)\right|_{\partial \Omega},\left.w\right|_{\partial \Omega}\right\rangle_{H^{-1 / 2}(\partial \Omega), H^{1 / 2}(\partial \Omega)},
\end{aligned}
$$

where

$$
w_{i}:=\frac{1}{\left|B_{i}\right|} \int_{B_{i}} w(y) \mathrm{d} y, \quad i=1, \ldots, N .
$$

Since this is true for all $w \in \mathcal{V}_{N}$, and therefore for all $\left(w_{1}, \ldots, w_{N}\right) \in \mathbf{R}^{N}$, one concludes that

$$
\beta_{i} \dot{T}_{i}-\left\langle\left.\kappa_{A} \frac{\partial T}{\partial n}\right|_{\partial B_{i}}, 1\right\rangle_{H^{-1 / 2}\left(\partial B_{i}\right), H^{1 / 2}\left(\partial B_{i}\right)}=0
$$

in $H^{-1}((0, \tau))$ for all $i=1, \ldots, N$, and

$$
\left.\kappa_{A} \frac{\partial T}{\partial n}\right|_{\partial \Omega}=0
$$

in $H_{00}^{1 / 2}((0, \tau) \times \partial \Omega)^{\prime}$.

Conversely, if $T$ satisfies (2.5), (2.6) and (2.7), the equality above shows that (2.4) holds. 
Proof of Proposition 2.3. Let $b$ be the bilinear form defined on $\mathcal{V}_{N} \times \mathcal{V}_{N}$ by

$$
b(u, v)=\int_{A} \kappa_{A}(x) \nabla_{x} u(x) \cdot \nabla_{x} v(x) \mathrm{d} x ;
$$

observe that

$$
|b(u, v)| \leq \frac{\kappa_{M}}{\rho_{m} C_{m}}(u \mid u)_{\mathcal{V}_{N}}^{1 / 2}(v \mid v)_{\mathcal{V}_{N}}^{1 / 2}
$$

while

$$
b(u, u) \geq \frac{\kappa_{m}}{\rho_{M} C_{M}}\left((u \mid u)_{\mathcal{V}_{N}}-(u \mid u)_{\mathcal{H}_{N}}\right) .
$$

By the same argument as in the proof of Proposition 2.1, for each $T^{i n} \in \mathcal{H}_{N}$, there exists a unique weak solution to (2.2), and this solution satisfies the energy identity in the statement of Proposition 2.3.

\section{Proof of Theorem 2.4}

We keep the notation used in the proof of Proposition 2.1, especially with the same definitions of $a, b, \mathcal{H}$ and $\mathcal{V}$.

For each $\eta>0$, the weak solution $T_{\eta}$ to (2.1) satisfies the energy identity

$$
\begin{aligned}
\frac{1}{2} \int_{\Omega} \rho(x) C(x) T_{\eta}(t, x)^{2} \mathrm{~d} x & +\int_{0}^{t} \int_{A} \kappa_{A}(x)\left|\nabla_{x} T_{\eta}(s, x)\right|^{2} \mathrm{~d} x \mathrm{~d} s+\frac{1}{\eta} \int_{0}^{t} \int_{B} \kappa_{B}(x)\left|\nabla_{x} T_{\eta}(s, x)\right|^{2} \mathrm{~d} x \mathrm{~d} s \\
& =\frac{1}{2} \int_{\Omega} \rho(x) C(x) T^{i n}(x)^{2} \mathrm{~d} x .
\end{aligned}
$$

Hence, for $\eta \in(0,1)$, one has

$$
\left|T_{\eta}(t, \cdot)\right|_{\mathcal{H}}^{2} \leq\left|T^{i n}\right|_{\mathcal{H}}^{2} \quad \text { and } \int_{0}^{\infty}\left|\nabla_{x} T_{\eta}(t, \cdot)\right|_{\mathcal{H}}^{2} \mathrm{~d} t \leq \frac{\rho_{M} C_{M}}{2 \kappa_{m}}\left|T^{i n}\right|_{\mathcal{H}}^{2}
$$

Applying the Banach-Alaoglu theorem shows that the family $T_{\eta}$ is relatively compact in $L^{\infty}([0,+\infty) ; \mathcal{H})$ weak-* and in $L^{2}([0, \tau] ; \mathcal{V})$ weak for each $\tau>0$. Let $T$ be a limit point of $T_{\eta}$; passing to the limit in the energy identity above shows that, by convexity and weak limit,

$$
\int_{0}^{\infty} \int_{B}\left|\nabla_{x} T(t, x)\right|^{2} \mathrm{~d} x \mathrm{~d} t=0
$$

Thus the function $x \mapsto T(t, x)$ is constant on $B_{i}$ for $i=1, \ldots, N$ for a.e. $t \geq 0$ and $T \in L^{\infty}\left([0,+\infty) ; \mathcal{H}_{N}\right) \cap$ $L^{2}\left(0, \tau ; \mathcal{V}_{N}\right)$.

Write the variational formulation of $(2.1)$ for a test function $w \in \mathcal{V}_{N} \subset \mathcal{V}$ :

$$
\frac{\mathrm{d}}{\mathrm{d} t}\left(T_{\eta} \mid w\right)_{\mathcal{H}}+a\left(T_{\eta}, w\right)=0 \quad \text { in } L^{2}([0, \tau]) \text { for all } \tau>0
$$

Passing to the limit in a subsequence of $T_{\eta}$ converging to $T$ in $L^{\infty}([0,+\infty) ; \mathcal{H})$ weak-* and in $L^{2}(0, \tau ; \mathcal{V})$ weak, one finds that

$$
\begin{aligned}
a\left(T_{\eta}, w\right) & =\int_{A} \kappa_{A}(x) \nabla_{x} T_{\eta}(t, x) \cdot \nabla w(x) \mathrm{d} x+\frac{1}{\eta} \int_{B} \kappa_{B}(x) \nabla_{x} T_{\eta}(t, x) \cdot \nabla w(x) \mathrm{d} x \\
& =\int_{A} \kappa_{A}(x) \nabla_{x} T_{\eta}(t, x) \cdot \nabla w(x) \mathrm{d} x \\
& \rightarrow \int_{A} \kappa_{A}(x) \nabla_{x} T(t, x) \cdot \nabla w(x) \mathrm{d} x=b(T, w) \text { weakly in } L^{2}([0, \tau])
\end{aligned}
$$


since $\nabla_{x} T_{\eta} \rightarrow \nabla_{x} T$ weakly in $L^{2}([0, \tau] \times \Omega)$. (The second equality above comes from the fact that $\nabla w=0$ on $B$ since $w \in \mathcal{V}_{N}$.) On the other hand, for each $w \in \mathcal{V}_{N}$

$$
\begin{aligned}
\int_{0}^{\tau}\left|\frac{\mathrm{d}}{\mathrm{d} t}\left(T_{\eta}(t, \cdot) \mid w\right)_{\mathcal{H}}\right|^{2} \mathrm{~d} t & =\int_{0}^{\tau}\left|a\left(T_{\eta}(t, \cdot), w\right)\right|^{2} \mathrm{~d} t \\
& =\int_{0}^{\tau}\left|\int_{A} \kappa_{A}(x) \nabla_{x} T_{\eta}(t, x) \cdot \nabla w(x) \mathrm{d} x\right|^{2} \mathrm{~d} t \\
& \leq \int_{0}^{\tau} \int_{A} \kappa_{A}(x)\left|\nabla_{x} T_{\eta}(t, x)\right|^{2} \mathrm{~d} x \mathrm{~d} t \int_{A} \kappa_{A}(x)|\nabla w(x)|^{2} \mathrm{~d} x \\
& \leq \frac{\kappa_{M}}{2 \rho_{m} C_{m}}\left|T^{i n}\right|_{\mathcal{H}}^{2}|w|_{\mathcal{V}}^{2}
\end{aligned}
$$

while

$$
\left(T_{\eta} \mid w\right)_{\mathcal{H}} \rightarrow(T \mid w)_{\mathcal{H}} \text { in } L^{\infty}([0,+\infty)) \text { weak-*. }
$$

Therefore, for each $w \in \mathcal{V}_{N}$, one has

$$
\frac{\mathrm{d}}{\mathrm{d} t}(T \mid w)_{\mathcal{H}}+b(T, w)=0 \quad \text { in } L^{2}([0, \tau]) \text { for all } \tau>0,
$$

which implies in particular that

$$
\rho C \partial_{t} T \in L^{2}\left(0, \tau ; \mathcal{V}_{N}^{\prime}\right)
$$

and therefore $T \in C_{b}\left([0,+\infty) ; \mathcal{H}_{N}\right)$ by statement (a) of Lemma A.1. Besides, by the Ascoli-Arzela theorem,

$$
\left(T_{\eta}(t, \cdot) \mid w\right)_{\mathcal{H}} \rightarrow(T(t, \cdot) \mid w)_{\mathcal{H}} \quad \text { uniformly in } t \in[0, \tau] \text { for all } \tau>0 .
$$

In particular

$$
\left(T_{\eta}(0, \cdot) \mid w\right)_{\mathcal{H}}=\left(T^{i n} \mid w\right)_{\mathcal{H}} \rightarrow(T(0, \cdot) \mid w)_{\mathcal{H}}
$$

so that

$$
T(0, \cdot)=T^{i n} .
$$

In other words $T$ is the weak solution to (2.2) with initial data $T^{i n}$ - the uniqueness of the weak solution following from Proposition 2.3. By compactness of the family $T_{\eta}$ and uniqueness of the limit point, we conclude that

$$
T_{\eta} \rightarrow T \text { in } L^{\infty}([0,+\infty) ; \mathcal{H}) \text { weak-* and in } L^{2}(0, \tau ; \mathcal{V}) \text { weak }
$$

as $\eta \rightarrow 0$.

The energy identities in Propositions 2.1 and 2.3 are recast in the form

$$
\begin{aligned}
\frac{1}{2} \int_{\Omega} \rho(x) C(x) T_{\eta}(t, x)^{2} \mathrm{~d} x+\int_{0}^{t} \int_{A} \kappa_{A}(x)\left|\nabla_{x} T_{\eta}(s, x)\right|^{2} \mathrm{~d} x \mathrm{~d} s+\frac{1}{\eta} \int_{0}^{t} \int_{B} \kappa_{B}(x)\left|\nabla_{x} T_{\eta}(s, x)\right|^{2} \mathrm{~d} x \mathrm{~d} s & \\
& =\frac{1}{2} \int_{\Omega} \rho(x) C(x) T^{i n}(x)^{2} \mathrm{~d} x
\end{aligned}
$$

and

$$
\frac{1}{2} \int_{\Omega} \rho(x) C(x) T(t, x)^{2} \mathrm{~d} x+\int_{0}^{t} \int_{A} \kappa_{A}(x)\left|\nabla_{x} T(s, x)\right|^{2} \mathrm{~d} x \mathrm{~d} s=\frac{1}{2} \int_{\Omega} \rho(x) C(x) T^{i n}(x)^{2} \mathrm{~d} x .
$$

(Notice that the condition $T^{i n} \in \mathcal{H}_{N}$ is essential in order that

$$
\frac{1}{2} \int_{\Omega} \rho(x) C(x) T^{i n}(x)^{2} \mathrm{~d} x=\frac{1}{2} \int_{A} \rho_{A}(x) C_{A}(x) T^{i n}(x)^{2} \mathrm{~d} x+\frac{1}{2} \sum_{i=1}^{N} \beta_{i}\left|T_{i}^{i n}\right|^{2} ;
$$


likewise

$$
\frac{1}{2} \int_{\Omega} \rho(x) C(x) T(t, x)^{2} \mathrm{~d} x=\frac{1}{2} \int_{A} \rho_{A}(x) C_{A}(x) T(t, x)^{2} \mathrm{~d} x+\frac{1}{2} \sum_{i=1}^{N} \beta_{i} T_{i}(t)^{2}
$$

since $T(t, \cdot) \in \mathcal{H}_{N}$ for all $t>0$.)

On the other hand, by convexity and weak convergence

$$
\frac{1}{2} \int_{\Omega} \rho(x) C(x) T(t, x)^{2} \mathrm{~d} x \leq \varliminf_{\eta \rightarrow 0^{+}} \frac{1}{2} \int_{\Omega} \rho(x) C(x) T_{\eta}(t, x)^{2} \mathrm{~d} x \text { for all } t>0,
$$

and

$$
\int_{0}^{t} \int_{A} \kappa_{A}(x)\left|\nabla_{x} T(s, x)\right|^{2} \mathrm{~d} x \mathrm{~d} s \leq \varliminf_{\eta \rightarrow 0^{+}} \int_{0}^{t} \int_{A} \kappa_{A}(x)\left|\nabla_{x} T_{\eta}(s, x)\right|^{2} \mathrm{~d} x \mathrm{~d} s .
$$

We conclude from the energy identities recalled above that

$$
\frac{1}{2} \int_{\Omega} \rho(x) C(x) T_{\eta}(t, x)^{2} \mathrm{~d} x \rightarrow \frac{1}{2} \int_{\Omega} \rho(x) C(x) T(t, x)^{2} \mathrm{~d} x \text { for all } t>0,
$$

while

$$
\left\{\begin{array}{l}
\int_{0}^{t} \int_{A} \kappa_{A}(x)\left|\nabla_{x} T_{\eta}(s, x)\right|^{2} \mathrm{~d} x \mathrm{~d} s \rightarrow \int_{0}^{t} \int_{A} \kappa_{A}(x)\left|\nabla_{x} T(s, x)\right|^{2} \mathrm{~d} x \mathrm{~d} s, \\
\frac{1}{\eta} \int_{0}^{t} \int_{B} \kappa_{B}(x)\left|\nabla_{x} T_{\eta}(s, x)\right|^{2} \mathrm{~d} x \mathrm{~d} s \rightarrow 0
\end{array}\right.
$$

for all $t>0$.

Therefore

$$
T_{\eta} \rightarrow T \text { and } \nabla_{x} T_{\eta} \rightarrow \nabla_{x} T \text { strongly in } L^{2}([0, \tau] \times \Omega)
$$

as $\eta \rightarrow 0$.

\section{Proof of Proposition 2.5}

Since

$$
\begin{array}{r}
\frac{\mathrm{d}}{\mathrm{d} t} \int_{\Omega} T(t, x) \phi(x) \mathrm{d} x+\sigma \int_{\Omega} \nabla_{x} T(t, x) \cdot \nabla \phi(x) \mathrm{d} x+4 \pi \sigma \int_{\Omega}(\varrho(x) T(t, x)-\vartheta(t, x)) \phi(x) \mathrm{d} x=0, \\
\frac{\mathrm{d}}{\mathrm{d} t} \int_{\Omega} \vartheta(t, x) \psi(x) \mathrm{d} x+4 \pi \sigma^{\prime} \int_{\Omega}(\vartheta(t, x)-\varrho(x) T(t, x)) \psi(x) \mathrm{d} x=0,
\end{array}
$$

with

$$
T \in L^{2}\left(0, \tau ; H^{1}(\Omega)\right) \quad \text { and } \vartheta \in L^{2}\left(0, \tau ; L^{2}(\Omega)\right),
$$

one has

$$
\left|\frac{\mathrm{d}}{\mathrm{d} t} \int_{\Omega} T(t, x) \phi(x) \mathrm{d} x\right| \leq(4 \pi+1) \sigma\left(\|\varrho\|_{L^{\infty}}\|T(t, \cdot)\|_{H^{1}(\Omega)}+\|\vartheta\|_{L^{2}(\Omega)}\right)\|\phi\|_{H^{1}(\Omega)}
$$

and

$$
\left|\frac{\mathrm{d}}{\mathrm{d} t} \int_{\Omega} \vartheta(t, x) \psi(x) \mathrm{d} x\right| \leq 4 \pi \sigma^{\prime}\left(\|\varrho\|_{L^{\infty}}\|T(t, \cdot)\|_{L^{2}(\Omega)}+\|\vartheta\|_{L^{2}(\Omega)}\right)\|\psi\|_{L^{2}(\Omega)},
$$

so that the linear functionals

$$
\phi \mapsto \frac{\mathrm{d}}{\mathrm{d} t} \int_{\Omega} T(t, x) \phi(x) \mathrm{d} x \quad \text { and } \psi \mapsto \frac{\mathrm{d}}{\mathrm{d} t} \int_{\Omega} \vartheta(t, x) \psi(x) \mathrm{d} x
$$


are continuous on $H^{1}(\Omega)$ and on $L^{2}(\Omega)$, respectively with values in $L^{2}([0, \tau])$. Therefore

$$
\partial_{t} T \in L^{2}\left(0, \tau ; H^{1}(\Omega)^{\prime}\right) \quad \text { and } \partial_{t} \vartheta \in L^{2}([0, \tau] \times \Omega)
$$

for each $\tau>0$. Since $T \in L^{2}\left(0, \tau ; H^{1}(\Omega)\right)$ and $\vartheta \in L^{2}\left(0, \tau ; L^{2}(\Omega)\right)$, this implies that

$$
T \text { and } \vartheta \in C_{b}\left(\mathbf{R}_{+} ; L^{2}(\Omega)\right) \text {. }
$$

Since the system (2.11) is linear, proving uniqueness reduces to proving that the only weak solution to (2.11) satisfying the initial condition $T^{i n}=\vartheta^{i n}=0$ is the trivial solution $T=\vartheta=0$.

By Lemma A.2, taking $\phi(x)=T(t, x)$ and $\psi(x)=\frac{\sigma}{\sigma^{\prime}} \vartheta(t, x) / \varrho(x)$, one has

$$
\begin{aligned}
\left\langle\partial_{t} T(t, \cdot), T(t, \cdot)\right\rangle_{H^{1}(\Omega)^{\prime}, H^{1}(\Omega)}+\sigma \int_{\Omega}\left|\nabla_{x} T(t, x)\right|^{2} \mathrm{~d} x+4 \pi \sigma \int_{\Omega}(\varrho(x) T(t, x)-\vartheta(t, x)) T(t, x) \mathrm{d} x & =0, \\
\frac{\sigma}{\sigma^{\prime}} \int_{\Omega} \frac{1}{\varrho(x)} \vartheta(t, x) \partial_{t} \vartheta(t, x) \mathrm{d} x+4 \pi \sigma \int_{\Omega}(\vartheta(t, x)-\varrho(x) T(t, x)) \frac{\vartheta(t, x)}{\varrho(x)} \mathrm{d} x & =0 .
\end{aligned}
$$

Adding both sides of the identities above, one finds that

$$
\left\langle\partial_{t} T(t, \cdot), T(t, \cdot)\right\rangle_{H^{1}(\Omega)^{\prime}, H^{1}(\Omega)}+\frac{\sigma}{\sigma^{\prime}} \int_{\Omega} \frac{1}{\varrho(x)} \vartheta(t, x) \partial_{t} \vartheta(t, x) \mathrm{d} x+\sigma \int_{\Omega}\left|\nabla_{x} T(t, x)\right|^{2} \mathrm{~d} x=0 .
$$

Integrating both sides of the identity above on $[0, t]$ and applying Lemma A.1 leads to

$$
\frac{1}{2} \int_{\Omega} T(t, x)^{2} \mathrm{~d} x+\frac{\sigma}{\sigma^{\prime}} \int_{\Omega} \frac{1}{\varrho(x)} \vartheta(t, x)^{2} \mathrm{~d} x+\sigma \int_{0}^{t} \int_{\Omega}\left|\nabla_{x} T(s, x)\right|^{2} \mathrm{~d} x \mathrm{~d} s=0
$$

so that $T=\vartheta=0$.

Specializing the variational formulation to $\phi, \psi \in C_{c}^{\infty}(\Omega)$ shows that $T$ and $\vartheta$ satisfy

$$
\left\{\begin{array}{l}
\partial_{t} T-\sigma \Delta_{x} T+4 \pi \sigma(\varrho T-\vartheta)=0, \\
\partial_{t} \vartheta+4 \pi \sigma^{\prime}(\vartheta-\varrho T)=0
\end{array}\right.
$$

in the sense of distributions on $(0,+\infty) \times \Omega$.

Finally, we apply Lemma A.3 to the vector field

$$
(t, x) \mapsto\left(\left(T(t, x)+\frac{\sigma}{\sigma^{\prime}} \vartheta(t, x)\right),-\sigma \nabla_{x} T(t, x)\right) .
$$

Indeed,

$$
T+\frac{\sigma}{\sigma^{\prime}} \vartheta \in C_{b}\left([0,+\infty] ; L^{2}(\Omega)\right) \quad \text { and } \nabla_{x} T \in L^{2}([0, \tau] \times \Omega)
$$

for each $\tau>0$. By linear combination of the two partial differential equations in (2.11), one has

$$
\partial_{t}\left(T+\frac{\sigma}{\sigma^{\prime}} \vartheta\right)+\operatorname{div}_{x}\left(-\sigma \nabla_{x} T\right)=0
$$

in the sense of distributions on $(0,+\infty) \times \Omega$, while

$$
\frac{\mathrm{d}}{\mathrm{d} t} \int_{\Omega}\left(T(t, x)+\frac{\sigma}{\sigma^{\prime}} \vartheta(t, x)\right) \phi(x) \mathrm{d} x-\sigma \int_{\Omega} \nabla_{x} T(t, x) \cdot \nabla \phi(x) \mathrm{d} x=0
$$

for each $\phi \in H^{1}(\Omega)$. Therefore

$$
\left.\frac{\partial T}{\partial n}\right|_{(0, \tau) \times \partial \Omega}=0
$$

in $H_{00}^{1 / 2}((0, \tau) \times \partial \Omega)^{\prime}$ for each $\tau>0$. 


\section{Proof of the Homogenization Limit}

Proof of Theorem 2.6. The proof is decomposed in several steps and involves several auxiliary lemmas whose proofs belong to the next section.

\section{Step 1: Uniform bounds.}

The energy identity for the scaled infinite conductivity problem is

$$
\frac{1}{2} \int_{A_{\epsilon}} T_{\epsilon}(t, x)^{2} \mathrm{~d} x+\frac{1}{2} \epsilon \sum_{i=1}^{N} \frac{\sigma}{\sigma^{\prime}} T_{i, \epsilon}(t)^{2}+\sigma \int_{0}^{t} \int_{A_{\epsilon}}\left|\nabla_{x} T_{\epsilon}(s, x)\right|^{2} \mathrm{~d} x \mathrm{~d} s=\frac{1}{2} \int_{A_{\epsilon}} T_{\epsilon}^{i n}(x)^{2} \mathrm{~d} x+\frac{1}{2} \epsilon \sum_{i=1}^{N} \frac{\sigma}{\sigma^{\prime}}\left|T_{i, \epsilon}^{i n}\right|^{2}
$$

for all $t \geq 0$ and $\epsilon>0$.

As a first consequence of this energy identity, the function $T_{\epsilon} \in C_{b}\left([0,+\infty) ; \mathcal{H}_{N}\right)$ satisfies the bounds

$$
\begin{aligned}
\left\|T_{\epsilon}(t, \cdot)\right\|_{\mathcal{H}_{N}}^{2} & =\int_{\Omega} \rho(x) C(x) T_{\epsilon}(t, x)^{2} \mathrm{~d} x \\
& =\rho_{A} C_{A} \int_{A_{\epsilon}} T_{\epsilon}(t, x)^{2} \mathrm{~d} x+\frac{4 \pi}{3} \epsilon^{3} \rho_{B} C_{B} \sum_{i=1}^{N} T_{i, \epsilon}(t)^{2} \\
& \leq \rho_{A} C_{A}\left(\int_{A_{\epsilon}} T_{\epsilon}^{i n}(x)^{2} \mathrm{~d} x+\epsilon \sum_{i=1}^{N} \frac{\sigma}{\sigma^{\prime}}\left|T_{i, \epsilon}^{i n}\right|^{2}\right) \leq \rho_{A} C_{A} C^{i n}
\end{aligned}
$$

and

$$
\sigma \int_{0}^{t} \int_{A_{\epsilon}}\left|\nabla_{x} T_{\epsilon}(s, x)\right|^{2} \mathrm{~d} x \mathrm{~d} s \leq \frac{1}{2} \int_{A_{\epsilon}} T_{\epsilon}^{i n}(x)^{2} \mathrm{~d} x+\frac{1}{2} \epsilon \sum_{i=1}^{N} \frac{\sigma}{\sigma^{\prime}}\left|T_{i, \epsilon}^{i n}\right|^{2} \leq \frac{1}{2} C^{i n}
$$

since $T_{\epsilon}(t, x)=T_{i, \epsilon}(t)$ for a.e. $x \in B\left(x_{i}, \epsilon\right)$ and all $i=1, \ldots, N$.

A second consequence of the same energy identity is that

$$
\epsilon \sum_{i=1}^{N} \frac{\sigma}{\sigma^{\prime}} T_{i, \epsilon}(t)^{2} \leq \int_{A_{\epsilon}} T_{\epsilon}^{i n}(x)^{2} \mathrm{~d} x+\epsilon \sum_{i=1}^{N} \frac{\sigma}{\sigma^{\prime}}\left|T_{i, \epsilon}^{i n}\right|^{2} \leq C^{i n}
$$

for all $t \in[0,+\infty)$ and $\epsilon>0$. To the weak solution $T_{\epsilon}$ to the scaled infinite conductivity problem we associate the empirical measure

$$
\mu_{\epsilon}(t, \mathrm{~d} x \mathrm{~d} \theta):=\frac{1}{N} \sum_{i=1}^{N} \delta_{x_{i}} \otimes \delta_{T_{i, \epsilon}(t)}, \quad N=1 / \epsilon .
$$

Accordingly, we denote

$$
\mu_{\epsilon}^{i n}(\mathrm{~d} x \mathrm{~d} \theta):=\frac{1}{N} \sum_{i=1}^{N} \delta_{x_{i}} \otimes \delta_{T_{i, \epsilon}^{i n}}
$$

The estimate above is recast as

$$
\iint_{\Omega \times \mathbf{R}} \theta^{2} \mu_{\epsilon}(t, \mathrm{~d} x \mathrm{~d} \theta)=\epsilon \sum_{i=1}^{N} T_{i, \epsilon}(t)^{2} \leq \frac{\sigma^{\prime}}{\sigma} C^{i n} .
$$

\section{Step 2: Compactness properties.}

These uniform bounds obviously imply that the family $T_{\epsilon}$ is relatively compact in $L^{\infty}\left([0,+\infty) ; L^{2}(\Omega)\right)$ weak-* and in $L^{2}\left(0, \tau ; H^{1}(\Omega)\right)$ weak for all $\tau>0$. Likewise the family $\left(1+\theta^{2}\right) \mu_{\epsilon}$ is relatively compact in 
$L^{\infty}\left([0,+\infty) ; \mathcal{M}_{b}(\Omega \times \mathbf{R})\right)$ viewed as the dual of the Banach space ${ }^{5} L^{1}\left([0,+\infty) ; C_{0}(\Omega \times \mathbf{R})\right)$ equipped with the weak-* topology.

Henceforth, we denote by $(T, \mu)$ a limit point of the family $\left(T_{\epsilon}, \mu_{\epsilon}\right)$ as $\epsilon \rightarrow 0$. Define

$$
\vartheta(t, \cdot):=\int_{\mathbf{R}} \theta \mu(t, \cdot, \mathrm{d} \theta)
$$

and observe that

$$
\varrho:=\int_{\mathbf{R}} \mu(t, \cdot, \mathrm{d} \theta)
$$

(Notice in particular that this equality shows that the integral on the right hand side is independent of the time variable $t$.) We already know from $(2.16)$ that $\varrho \in C_{b}(\bar{\Omega})$ is a positive probability density, and we shall see shortly that $\vartheta \in L^{\infty}\left(\mathbf{R}_{+} ; L^{2}(\Omega)\right)$.

Next we return to the energy identity in Step 1 recast as follows

$$
\begin{aligned}
\frac{1}{2} \int_{\Omega} T_{\epsilon}(t, x)^{2} \mathrm{~d} x+\left(\frac{\sigma}{\sigma^{\prime}}-\frac{4}{3} \pi \epsilon^{2}\right) \iint_{\Omega \times \mathbf{R}} \frac{1}{2} \theta^{2} \mu_{\epsilon}(t, \mathrm{~d} x \mathrm{~d} \theta)+\sigma \int_{0}^{t} \int_{A_{\epsilon}}\left|\nabla_{x} T_{\epsilon}(s, x)\right|^{2} \mathrm{~d} x \mathrm{~d} s & =\frac{1}{2} \int_{A_{\epsilon}} T_{\epsilon}^{i n}(x)^{2} \mathrm{~d} x+\frac{1}{2} \epsilon \sum_{i=1}^{N} \frac{\sigma}{\sigma^{\prime}}\left|T_{i, \epsilon}^{i n}\right|^{2},
\end{aligned}
$$

so that

$$
\left(\frac{\sigma}{\sigma^{\prime}}-\frac{4}{3} \pi \epsilon^{2}\right) \iint_{\Omega \times \mathbf{R}} \theta^{2} \mu_{\epsilon}(t, \mathrm{~d} x \mathrm{~d} \theta) \leq C^{i n} .
$$

Thus, for each $R>0$, using $(x, \theta) \mapsto \min \left(\theta^{2}, R\right)$ as test function and the weak-* convergence of the family of measures $\left(1+|x|^{2}+\theta^{2}\right) \mu_{\epsilon}$, passing to the limit in each side of the inequality above, we get

$$
\frac{\sigma}{\sigma^{\prime}} \iint_{\Omega \times \mathbf{R}} \min \left(\theta^{2}, R\right) \mu(t, \mathrm{~d} x \mathrm{~d} \theta) \leq C^{i n} .
$$

Letting $R \rightarrow+\infty$, by monotone convergence

$$
\frac{\sigma}{\sigma^{\prime}} \iint_{\Omega \times \mathbf{R}} \theta^{2} \mu(t, \mathrm{~d} x \mathrm{~d} \theta) \leq C^{i n} .
$$

By the Cauchy-Schwarz inequality

$$
\vartheta(t, \cdot)^{2}=\left(\int_{\mathbf{R}} \theta \mu(t, \cdot, \mathrm{d} \theta)\right)^{2} \leq \int_{\mathbf{R}} \mu(t, \cdot, \mathrm{d} \theta) \int_{\mathbf{R}} \theta^{2} \mu(t, \cdot, \mathrm{d} \theta)=\varrho \int_{\mathbf{R}} \theta^{2} \mu(t, \cdot, \mathrm{d} \theta)
$$

so that

$$
\int_{\Omega} \vartheta(t, x)^{2} \mathrm{~d} x \leq \int_{\Omega} \varrho(x) \int_{\mathbf{R}} \theta^{2} \mu(t, \mathrm{~d} x \mathrm{~d} \theta) \leq \frac{\sigma^{\prime}}{\sigma} C^{i n}\|\varrho\|_{L^{\infty}(\Omega)},
$$

by (2.16). This implies in particular that $\vartheta \in L^{\infty}\left(\mathbf{R}_{+} ; L^{2}(\Omega)\right)$ as announced.

Thus, going back to (6.1), we conclude that, for each $\tau>0$,

$$
T \in L^{\infty}\left([0,+\infty) ; L^{2}(\Omega)\right) \cap L^{2}\left(0, \tau ; H^{1}(\Omega)\right) \quad \text { and } \vartheta \in L^{\infty}\left([0,+\infty) ; L^{2}(\Omega)\right) .
$$

\footnotetext{
${ }^{5}$ If $X$ is a locally compact space, the notation $C_{0}(X)$ designates the set of real-valued continuous functions $f$ defined on $X$ such that $f$ converges to 0 at infinity. This is a Banach space for the norm $\|f\|=\sup _{x \in X}|f(x)|$.
} 


\section{Step 3: Passing to the limit in the variational formulation.}

Start from the variational formulation of the scaled infinite conductivity problem: for each $\Phi_{\epsilon} \in \mathcal{V}_{N}$

$$
\frac{\mathrm{d}}{\mathrm{d} t}\left(\int_{A_{\epsilon}} T_{\epsilon}(t, x) \Phi_{\epsilon}(x) \mathrm{d} x+\frac{3 \sigma}{4 \pi \sigma^{\prime}} \frac{1}{\epsilon^{2}} \int_{B_{\epsilon}} T_{\epsilon}(t, x) \Phi_{\epsilon}(x) \mathrm{d} x\right)+\sigma \int_{A_{\epsilon}} \nabla_{x} T_{\epsilon}(t, x) \cdot \nabla \Phi_{\epsilon}(x) \mathrm{d} x=0
$$

for a.e. $t \in[0,+\infty)$.

Since $T_{\epsilon}(t, \cdot) \in \mathcal{V}_{N}$, assuming that $\Phi_{\epsilon} \in \mathcal{V}_{N} \cap C_{b}(\bar{\Omega})$,

$$
\frac{3 \sigma}{4 \pi \sigma^{\prime}} \frac{1}{\epsilon^{2}} \int_{B_{\epsilon}} T_{\epsilon}(t, x) \Phi_{\epsilon}(x) \mathrm{d} x=\frac{\sigma}{\sigma^{\prime}} \epsilon \sum_{i=1}^{N} T_{i, \epsilon}(t) \Phi_{i, \epsilon}=\frac{\sigma}{\sigma^{\prime}} \iint_{\Omega \times \mathbf{R}} \Phi_{\epsilon}(x) \theta \mu_{\epsilon}(t, \mathrm{~d} x \mathrm{~d} \theta) .
$$

On the other hand

$$
\begin{aligned}
\int_{A_{\epsilon}} T_{\epsilon}(t, x) \Phi_{\epsilon}(x) \mathrm{d} x & =\int_{\Omega} T_{\epsilon}(t, x) \Phi_{\epsilon}(x) \mathrm{d} x-\int_{B_{\epsilon}} T_{\epsilon}(t, x) \Phi_{\epsilon}(x) \mathrm{d} x \\
& =\int_{\Omega} T_{\epsilon}(t, x) \Phi_{\epsilon}(x) \mathrm{d} x-\frac{4 \pi}{3} \epsilon^{3} \sum_{i=1}^{N} T_{i, \epsilon}(t) \Phi_{i, \epsilon}(t) \\
& =\int_{\Omega} T_{\epsilon}(t, x) \Phi_{\epsilon}(x) \mathrm{d} x-\frac{4 \pi}{3} \epsilon^{2} \iint_{\Omega \times \mathbf{R}} \Phi_{\epsilon}(x) \theta \mu_{\epsilon}(t, \mathrm{~d} x \mathrm{~d} \theta)
\end{aligned}
$$

so that

$$
\begin{aligned}
\left|\int_{\Omega} T_{\epsilon}(t, x) \Phi_{\epsilon}(x) \mathrm{d} x-\int_{A_{\epsilon}} T_{\epsilon}(t, x) \Phi_{\epsilon}(x) \mathrm{d} x\right| & \leq \frac{2 \pi}{3} \epsilon^{2}\left\|\Phi_{\epsilon}\right\|_{L^{\infty}(\Omega)} \iint_{\Omega \times \mathbf{R}}\left(1+\theta^{2}\right) \mu_{\epsilon}(t, \mathrm{~d} x \mathrm{~d} \theta) \\
& \leq \frac{2 \pi}{3} \epsilon^{2}\left\|\Phi_{\epsilon}\right\|_{L^{\infty}(\Omega)}\left(1+\frac{\sigma^{\prime}}{\sigma} C^{i n}\right) .
\end{aligned}
$$

Finally

$$
\int_{A_{\epsilon}} \nabla_{x} T_{\epsilon}(t, x) \cdot \nabla \Phi_{\epsilon}(x) \mathrm{d} x=\int_{\Omega} \nabla_{x} T_{\epsilon}(t, x) \cdot \nabla \Phi_{\epsilon}(x) \mathrm{d} x .
$$

We shall pass to the limit in the variational formulation above for two different classes of test functions $\Phi_{\epsilon}$.

\section{Step 4: First class of test functions.}

Let $\phi \in C_{c}^{1}(\bar{\Omega})$. By the mean value theorem

$$
\left|\phi(x)-\phi\left(x_{i}\right)\right| \leq \epsilon\|D \phi\|_{L^{\infty}} \quad \text { for all } x \in B\left(x_{i}, \epsilon\right),
$$

so that $\phi$ "almost" belongs to $\mathcal{V}_{N}$ - but in general does not belong to $\mathcal{V}_{N}$. This difficulty is fixed by the following procedure.

For each $\psi \in C(\overline{B(0, \epsilon)})$, define $\chi[\psi]$ to be the solution of

$$
\begin{cases}\Delta \chi[\psi](z)=0, & \epsilon<|z|<r_{\epsilon}, \\ \chi[\psi](z)=\psi(z), & |z| \leq \epsilon, \\ \chi[\psi](z)=0, & |z|=r_{\epsilon} .\end{cases}
$$

Define

$$
\mathcal{Q}_{\epsilon}(x):=\sum_{i=1}^{N} \chi\left[\phi\left(x_{i}+\cdot\right)-\phi\left(x_{i}\right)\right]\left(x-x_{i}\right),
$$

where the notation $\phi\left(x_{i}+\cdot\right)$ designates the function $y \mapsto \phi\left(x_{i}+y\right)$, and let

$$
\Phi_{\epsilon}(x):=\phi(x)-\mathcal{Q}_{\epsilon}(x) .
$$


Lemma 6.1. For each $\epsilon>0$, one has

$$
\left\|\mathcal{Q}_{\epsilon}\right\|_{L^{\infty}(\Omega)} \leq 2\|\phi\|_{L^{\infty}(\Omega)}
$$

Besides

$$
\mathcal{Q}_{\epsilon} \rightarrow 0 \text { in } H^{1}(\Omega) \text { strong }
$$

as $\epsilon \rightarrow 0$.

The proof of this lemma is postponed to the end of this section. Taking this for granted, one has

$$
\Phi_{\epsilon} \rightarrow \phi \text { in } H^{1}(\Omega) \text { strong }
$$

as $\epsilon \rightarrow 0$. Therefore

$$
\begin{aligned}
\int_{A_{\epsilon}} \nabla_{x} T_{\epsilon}(t, x) \cdot \nabla \Phi_{\epsilon}(x) \mathrm{d} x & =\int_{\Omega} \nabla_{x} T_{\epsilon}(t, x) \cdot \nabla \Phi_{\epsilon}(x) \mathrm{d} x \\
& \rightarrow \int_{\Omega} \nabla_{x} T(t, x) \cdot \nabla \phi(x) \mathrm{d} x \text { weakly in } L^{2}([0,+\infty))
\end{aligned}
$$

as $\epsilon \rightarrow 0$.

On the other hand

$$
\begin{aligned}
\int_{\Omega} T_{\epsilon}(t, x) \Phi_{\epsilon}(x) \mathrm{d} x & =\int_{\Omega} T_{\epsilon}(t, x) \phi(x) \mathrm{d} x-\int_{\Omega} T_{\epsilon}(t, x) \mathcal{Q}_{\epsilon}(x) \mathrm{d} x \\
& \rightarrow \int_{\Omega} T(t, x) \phi(x) \mathrm{d} x \text { in } L^{\infty}([0,+\infty)) \text { weak-* }
\end{aligned}
$$

as $\epsilon \rightarrow 0$ since

$$
\left|\int_{\Omega} T_{\epsilon}(t, x) \mathcal{Q}_{\epsilon}(x) \mathrm{d} x\right| \leq\left\|T_{\epsilon}(t, \cdot)\right\|_{L^{2}}\left\|\mathcal{Q}_{\epsilon}\right\|_{L^{2}}
$$

Indeed

$$
\sup _{t \geq 0}\left\|T_{\epsilon}(t, \cdot)\right\|_{L^{2}}<\infty, \quad \text { while }\left\|\mathcal{Q}_{\epsilon}\right\|_{L^{2}} \rightarrow 0 \text { as } \epsilon \rightarrow 0
$$

by Lemma 6.1 .

Finally

$$
\iint_{\Omega \times \mathbf{R}} \Phi_{\epsilon}(x) \theta \mu_{\epsilon}(t, \mathrm{~d} x \mathrm{~d} \theta)=\iint_{\Omega \times \mathbf{R}} \phi(x) \theta \mu_{\epsilon}(t, \mathrm{~d} x \mathrm{~d} \theta)
$$

since $\phi\left(x_{i}\right)=\Phi_{\epsilon}\left(x_{i}\right)$ for $i=1, \ldots, N$, so that

$$
\iint_{\Omega \times \mathbf{R}} \Phi_{\epsilon}(x) \theta \mu_{\epsilon}(t, \mathrm{~d} x \mathrm{~d} \theta) \rightarrow \iint_{\Omega \times \mathbf{R}} \phi(x) \theta \mu(t, \mathrm{~d} x \mathrm{~d} \theta) \text { in } L^{\infty}([0,+\infty)) \text { weak-* }
$$

as $\epsilon \rightarrow 0$.

By construction $\Phi_{\epsilon} \in \mathcal{V}_{N}$, so that $\Phi_{\epsilon}$ can be used as a test function in the variational formulation. Passing to the limit in the variational formulation of the scaled infinite heat conductivity problem in the sense of distributions gives

$$
\frac{\mathrm{d}}{\mathrm{d} t}\left(\int_{\Omega} T(t, x) \phi(x) \mathrm{d} x+\frac{\sigma}{\sigma^{\prime}} \iint_{\Omega \times \mathbf{R}} \phi(x) \theta \mu(t, \mathrm{~d} x \mathrm{~d} \theta)\right)+\sigma \int_{\Omega} \nabla_{x} T(t, x) \cdot \nabla \phi(x) \mathrm{d} x=0
$$

in $L_{l o c}^{2}([0,+\infty))$ for each $\phi \in C_{c}^{1}(\bar{\Omega})$. 


\section{Step 5: Second class of test functions}

In this step, we shall use a class of test functions $\Psi_{\epsilon} \in H^{1}(\Omega)$ such that $\left.\Psi_{\epsilon}\right|_{B\left(x_{i}, \epsilon\right)}=0$ for all $i=1, \ldots, N$. Given $\phi \in C_{c}^{1}(\bar{\Omega})$, define $\Psi_{\epsilon}$ as follows:

$$
\Psi_{\epsilon}(x):=\phi(x)-\mathcal{P}_{\epsilon}(x)
$$

where

$$
\mathcal{P}_{\epsilon}(x):=\sum_{i=1}^{N} \chi\left[\phi\left(x_{i}+\cdot\right)\right]\left(x-x_{i}\right) .
$$

We shall further decompose $\mathcal{P}_{\epsilon}$ as follows:

$$
\begin{aligned}
\mathcal{P}_{\epsilon}(x) & =\sum_{i=1}^{N} \chi\left[\phi\left(x_{i}+\cdot\right)-\phi\left(x_{i}\right)\right]\left(x-x_{i}\right)+\sum_{i=1}^{N} \chi\left[\phi\left(x_{i}\right)\right]\left(x-x_{i}\right) \\
& =\mathcal{Q}_{\epsilon}(x)+\mathcal{R}_{\epsilon}(x) .
\end{aligned}
$$

Likewise, one associates to the solution $T_{\epsilon}$ to the scaled infinite heat conductivity problem

$$
\Theta_{\epsilon}(t, x):=T_{\epsilon}(t, x)-\mathcal{S}_{\epsilon}(t, x)
$$

where

$$
\mathcal{S}_{\epsilon}(t, x):=\sum_{i=1}^{N} \chi\left[T_{i, \epsilon}(t)\right]\left(x-x_{i}\right) .
$$

The variational formulation for the test function $\Psi_{\epsilon}$ becomes

$$
\frac{\mathrm{d}}{\mathrm{d} t} \int_{\Omega} T_{\epsilon}(t, x) \Psi_{\epsilon}(x) \mathrm{d} x+\sigma \int_{\Omega} \nabla_{x} T_{\epsilon}(t, x) \cdot \nabla \Psi_{\epsilon}(x) \mathrm{d} x=0
$$

in $L_{l o c}^{2}([0,+\infty))$, since $\Psi_{\epsilon}=0$ on $B\left(x_{i}, \epsilon\right)$ for all $i=1, \ldots, N$.

Lemma 6.2. One has

$$
\mathcal{R}_{\epsilon} \rightarrow 0 \quad \text { in } H^{1}(\Omega) \text { weak }
$$

so that

$$
\mathcal{P}_{\epsilon} \rightarrow 0 \text { in } H^{1}(\Omega) \text { weak }
$$

while

$$
\mathcal{S}_{\epsilon} \rightarrow 0 \quad \text { in } L^{\infty}\left([0,+\infty] ; H^{1}(\Omega)\right) \text { weak-* }
$$

as $\epsilon \rightarrow 0$.

Taking this lemma for granted, and observing that

$$
\operatorname{supp}\left(\mathcal{P}_{\epsilon}\right) \subset \operatorname{supp}(\phi)+\overline{B\left(0, r_{\epsilon}\right)},
$$

the Rellich compactness theorem implies that

$$
\mathcal{P}_{\epsilon} \rightarrow 0 \quad \text { in } L^{2}(\Omega) \text { strong }
$$

as $\epsilon \rightarrow 0$, so that

$$
\int_{\Omega} T_{\epsilon}(t, x) \Psi_{\epsilon}(x) \mathrm{d} x \rightarrow \int_{\Omega} T(t, x) \phi(x) \mathrm{d} x \text { in } L^{\infty}([0,+\infty)) \text { weak-* }
$$

as $\epsilon \rightarrow 0$. 
Next, decompose

$$
\begin{aligned}
\int_{\Omega} \nabla_{x} T_{\epsilon}(t, x) \cdot \nabla \Psi_{\epsilon}(x) \mathrm{d} x= & \int_{\Omega} \nabla_{x} \Theta_{\epsilon}(t, x) \cdot \nabla \phi(x) \mathrm{d} x \\
& -\int_{\Omega} \nabla_{x} \Theta_{\epsilon}(t, x) \cdot \nabla \mathcal{P}_{\epsilon}(x) \mathrm{d} x \\
& +\int_{\Omega} \nabla_{x} \mathcal{S}_{\epsilon}(t, x) \cdot \nabla \Psi_{\epsilon}(x) \mathrm{d} x .
\end{aligned}
$$

Since $\mathcal{S}_{\epsilon} \rightarrow 0$ in $L^{2}\left(0, \tau ; H_{0}^{1}(\Omega)\right)$ weak as $\epsilon \rightarrow 0$, one has

$$
\nabla_{x} \Theta_{\epsilon}=\nabla_{x} T_{\epsilon}-\nabla_{x} \mathcal{S}_{\epsilon} \rightarrow \nabla_{x} T \text { in } L^{2}([0, \tau] \times \Omega) \text { weak }
$$

as $\epsilon \rightarrow 0$, so that

$$
\int_{\Omega} \nabla_{x} \Theta_{\epsilon}(t, x) \cdot \nabla \phi(x) \mathrm{d} x \rightarrow \int_{\Omega} \nabla_{x} T(t, x) \cdot \nabla \phi(x) \mathrm{d} x \text { in } L^{2}([0, \tau]) \text { weak }
$$

as $\epsilon \rightarrow 0$.

Furthermore, one has

$$
\begin{aligned}
\int_{\Omega} \nabla_{x} \mathcal{S}_{\epsilon}(t, x) \cdot \nabla \Psi_{\epsilon}(x) \mathrm{d} x= & \int_{\Omega} \nabla_{x} \mathcal{S}_{\epsilon}(t, x) \cdot \nabla \phi(x) \mathrm{d} x-\int_{\Omega} \nabla_{x} \mathcal{S}_{\epsilon}(t, x) \cdot \nabla \mathcal{Q}_{\epsilon}(x) \mathrm{d} x \\
& -\sum_{i=1}^{N} \int_{B\left(x_{i}, r_{\epsilon}\right) \backslash B\left(x_{i}, \epsilon\right)} \nabla \chi\left[T_{i, \epsilon}(t)\right](z) \cdot \nabla \chi\left[\phi\left(x_{i}\right)\right](z) \mathrm{d} z .
\end{aligned}
$$

As noticed above, $\nabla_{x} \mathcal{S}_{\epsilon} \rightarrow 0$ weakly in $L^{2}([0, \tau] \times \Omega)$ for all $\tau>0$ as $\epsilon \rightarrow 0$, and therefore

$$
\int_{\Omega} \nabla_{x} \mathcal{S}_{\epsilon}(t, x) \cdot \nabla \phi(x) \mathrm{d} x \rightarrow 0 \quad \text { in } L^{2}([0, \tau]) \text { weak }
$$

for all $\tau>0$ as $\epsilon \rightarrow 0$, while

$$
\int_{\Omega} \nabla_{x} \mathcal{S}_{\epsilon}(t, x) \cdot \nabla \mathcal{Q}_{\epsilon}(x) \mathrm{d} x \rightarrow 0 \quad \text { in } L^{2}([0, \tau]) \text { strong }
$$

for all $\tau>0$ as $\epsilon \rightarrow 0$ by Lemma 6.1 .

The third term on the right hand side of the last equality is handled with the following lemma.

Lemma 6.3. One has

$$
\sum_{i=1}^{N} \int_{B\left(0, r_{\epsilon}\right) \backslash B(0, \epsilon)} \nabla \chi\left[T_{i, \epsilon}(t)\right](z) \cdot \nabla \chi\left[\phi\left(x_{i}\right)\right](z) \mathrm{d} z \rightarrow 4 \pi \int_{\Omega \times \mathbf{R}} \phi(x) \theta \mu(t, \mathrm{~d} x \mathrm{~d} \theta)
$$

in $L^{\infty}\left(\mathbf{R}_{+}\right)$weak-**

Therefore

$$
\int_{\Omega} \nabla_{x} \mathcal{S}_{\epsilon}(t, x) \cdot \nabla \Psi_{\epsilon}(x) \mathrm{d} x \rightarrow-4 \pi \iint_{\Omega \times \mathbf{R}} \phi(x) \theta \mu(t, \mathrm{~d} x \mathrm{~d} \theta)
$$

in $L^{2}([0, \tau])$ weak as $\epsilon \rightarrow 0$.

It remains to treat the term

$$
\int_{\Omega} \nabla_{x} \Theta_{\epsilon}(t, x) \cdot \nabla \mathcal{P}_{\epsilon}(x) \mathrm{d} x=\int_{\Omega} \nabla_{x} \Theta_{\epsilon}(t, x) \cdot \nabla \mathcal{Q}_{\epsilon}(x) \mathrm{d} x+\sum_{i=1}^{N} \int_{B\left(x_{i}, r_{\epsilon}\right) \backslash B\left(x_{i}, \epsilon\right)} \nabla_{x} \Theta_{\epsilon}(t, z) \cdot \nabla \chi\left[\phi\left(x_{i}\right)\right](z) \mathrm{d} z .
$$


By Lemma 6.1, $\mathcal{Q}_{\epsilon} \rightarrow 0$ in $H^{1}(\Omega)$ strong; by the second convergence in Lemma 6.2 , the family $\nabla_{x} \mathcal{S}_{\epsilon}$ is bounded in $L^{2}([0, \tau] \times \Omega)$ for each $\tau>0$, while $\nabla_{x} T_{\epsilon}$ is bounded in $L^{2}([0, \tau] \times \Omega)$ as explained in Step 1 . Thus $\nabla_{x} \Theta_{\epsilon}$ is bounded in $L^{2}([0, \tau] \times \Omega)$ for all $\tau>0$, so that

$$
\int_{\Omega} \nabla_{x} \Theta_{\epsilon}(t, x) \cdot \nabla \mathcal{Q}_{\epsilon}(x) \mathrm{d} x \rightarrow 0 \text { in } L^{2}([0, \tau]) \text { strong }
$$

for each $\tau>0$ as $\epsilon \rightarrow 0$.

Next, by Green's formula

$$
\int_{B\left(x_{i}, r_{\epsilon}\right) \backslash B\left(x_{i}, \epsilon\right)} \nabla_{x} \Theta_{\epsilon}(t, z) \cdot \nabla \chi\left[\phi\left(x_{i}\right)\right](z) \mathrm{d} z=\int_{\partial B\left(x_{i}, r_{\epsilon}\right)} \Theta_{\epsilon}(t, z) \frac{\partial \chi\left[\phi\left(x_{i}\right)\right]}{\partial n}(z) \mathrm{d} z
$$

since $\chi\left[\phi\left(x_{i}\right)\right]$ is harmonic on $B\left(x_{i}, r_{\epsilon}\right) \backslash B\left(x_{i}, \epsilon\right)$ and $\left.\Theta_{\epsilon}\right|_{\partial B\left(x_{i}, \epsilon\right)}=0$.

Lemma 6.4. For each $\phi \in C_{b}\left(\mathbf{R}^{3}\right)$, one has

$$
\sum_{i=1}^{N} \frac{\partial}{\partial n}\left(\chi\left[\phi\left(x_{i}\right)\right]\left(x-x_{i}\right)\right) \delta_{\partial B\left(x_{i}, r_{\epsilon}\right)}=-\frac{\epsilon r_{\epsilon}}{r_{\epsilon}^{2}\left(r_{\epsilon}-\epsilon\right)} \sum_{i=1}^{N} \phi\left(x_{i}\right) \delta_{\partial B\left(x_{i}, r_{\epsilon}\right)} \rightarrow-4 \pi \varrho \phi
$$

in $H^{-1}\left(\mathbf{R}^{3}\right)$. We recall that $\varrho \in C_{b}(\bar{\Omega})$ is defined as follows:

$$
\frac{1}{N} \sum_{i=1}^{N} \delta_{x_{i}} \rightarrow \varrho \mathscr{L}^{3}
$$

weakly in the sense of probability measures on $\bar{\Omega}$, where $\mathscr{L}^{3}$ designates the 3 -dimensional Lebesgue measure.

Taking this lemma for granted, we see that

$$
\int_{\Omega} \nabla_{x} \Theta_{\epsilon}(t, x) \cdot \nabla \mathcal{R}_{\epsilon}(x) \mathrm{d} x \rightarrow-4 \pi \int_{\Omega} \varrho(x) T(t, x) \phi(x) \mathrm{d} x \text { in } L^{2}([0, \tau]) \text { weak }
$$

for each $\tau>0$ as $\epsilon \rightarrow 0$.

Summarizing the various limits established in this step, we conclude that, for each $\phi \in C_{c}^{1}(\bar{\Omega})$

$$
\frac{\mathrm{d}}{\mathrm{d} t} \int_{\Omega} T(t, x) \phi(x) \mathrm{d} x+\sigma \int_{\Omega} \nabla_{x} T(t, x) \cdot \nabla \phi(x) \mathrm{d} x+4 \pi \sigma \int_{\Omega} \varrho(x) T(t, x) \phi(x) \mathrm{d} x-4 \pi \sigma \iint_{\Omega \times \mathbf{R}} \phi(x) \theta \mu(t, \mathrm{~d} x \mathrm{~d} \theta)=0
$$

in $L_{l o c}^{2}([0,+\infty))$.

\section{Step 6: Initial conditions}

As explained in Steps 3-4, for each $\phi \in C_{c}^{1}(\bar{\Omega})$, defining

$$
\Phi_{\epsilon}=\phi-\mathcal{Q}_{\epsilon} \in \mathcal{V}_{N}
$$

one has

$$
\int_{A_{\epsilon}} T_{\epsilon}(t, x) \Phi_{\epsilon}(x) \mathrm{d} x+\frac{\sigma}{\sigma^{\prime}} \iint_{\Omega \times \mathbf{R}} \Phi_{\epsilon}(x) \theta \mu_{\epsilon}(t, \mathrm{~d} x \mathrm{~d} \theta) \rightarrow \int_{\Omega} T(t, x) \phi(x) \mathrm{d} x+\frac{\sigma}{\sigma^{\prime}} \iint_{\Omega \times \mathbf{R}} \phi(x) \theta \mu(t, \mathrm{~d} x \mathrm{~d} \theta)
$$

in $L^{2}([0, \tau])$ weak as $\epsilon \rightarrow 0$, while

$$
\begin{gathered}
\int_{0}^{\infty}\left|\frac{\mathrm{d}}{\mathrm{d} t}\left(\int_{A_{\epsilon}} T_{\epsilon}(t, x) \Phi_{\epsilon}(x) \mathrm{d} x+\frac{\sigma}{\sigma^{\prime}} \iint_{\Omega \times \mathbf{R}} \Phi_{\epsilon}(x) \theta \mu_{\epsilon}(t, \mathrm{~d} x \mathrm{~d} \theta)\right)\right|^{2} \mathrm{~d} t \\
\leq \sigma^{2}\left\|\nabla \Phi_{\epsilon}\right\|_{L^{2}(\Omega)}^{2} \int_{0}^{\infty}\left\|\nabla_{x} T_{\epsilon}(t, \cdot)\right\|_{L^{2}(\Omega)}^{2} \mathrm{~d} t \\
\leq C^{i n} \sigma\left(\|\nabla \phi\|_{L^{2}(\Omega)}+o(1)\right)^{2} .
\end{gathered}
$$


By Ascoli-Arzela's theorem, the convergence in (6.5) is uniform on $[0, \tau]$ for each $\tau$. In particular

$$
\begin{aligned}
\int_{A_{\epsilon}} T_{\epsilon}^{i n}(x) \Phi_{\epsilon}(x) \mathrm{d} x+\frac{\sigma}{\sigma^{\prime}} \iint_{\Omega \times \mathbf{R}} \Phi_{\epsilon}(x) \theta \mu_{\epsilon}^{i n}(\mathrm{~d} x \mathrm{~d} \theta) & \rightarrow \int_{\Omega} T^{i n}(x) \phi(x) \mathrm{d} x+\frac{\sigma}{\sigma^{\prime}} \iint_{\Omega \times \mathbf{R}} \phi(x) \theta \mu^{i n}(\mathrm{~d} x \mathrm{~d} \theta) \\
& =\int_{\Omega} T(0, x) \phi(x) \mathrm{d} x+\frac{\sigma}{\sigma^{\prime}} \iint_{\Omega \times \mathbf{R}} \phi(x) \theta \mu(0, \mathrm{~d} x \mathrm{~d} \theta)
\end{aligned}
$$

for each $\phi \in C_{c}^{1}(\bar{\Omega})$, so that

$$
T(0, \cdot)+\frac{\sigma^{\prime}}{\sigma} \int_{\mathbf{R}} \theta \mu(0, \cdot, \mathrm{d} \theta)=T^{i n}+\frac{\sigma^{\prime}}{\sigma} \int_{\mathbf{R}} \theta \mu^{i n}(\cdot, \mathrm{d} \theta) .
$$

Likewise, we have seen in Step 5 that, for each $\phi \in C_{c}^{1}(\bar{\Omega})$, defining $\Psi_{\epsilon}$ as

$$
\Psi_{\epsilon}=\phi-\mathcal{P}_{\epsilon}
$$

one has

$$
\int_{\Omega} T_{\epsilon}(t, x) \Psi_{\epsilon}(x) \mathrm{d} x \rightarrow \int_{\Omega} T(t, x) \phi(x) \mathrm{d} x \text { in } L^{\infty}([0,+\infty)) \text { weak-* }
$$

as $\epsilon \rightarrow 0$. Besides

$$
\begin{aligned}
\int_{0}^{\infty}\left|\frac{\mathrm{d}}{\mathrm{d} t} \int_{\Omega} T_{\epsilon}(t, x) \Psi_{\epsilon}(x) \mathrm{d} x\right|^{2} \mathrm{~d} t & \leq \sigma^{2}\left\|\nabla \Psi_{\epsilon}\right\|_{L^{2}(\Omega)}^{2} \int_{0}^{\infty} \int_{\Omega}\left|\nabla_{x} T_{\epsilon}(t, x)\right|^{2} \mathrm{~d} x \mathrm{~d} t \\
& \leq C^{i n} \sigma\left(\|\nabla \phi\|_{L^{2}(\Omega)}+o(1)\right)^{2}
\end{aligned}
$$

By the Ascoli-Arzela theorem, the convergence in (6.6) is uniform in $[0, \tau]$ for each $\tau>0$. In particular

$$
\int_{\Omega} T_{\epsilon}^{i n}(x) \Psi_{\epsilon}(x) \mathrm{d} x \rightarrow \int_{\Omega} T^{i n}(x) \phi(x) \mathrm{d} x=\int_{\Omega} T(0, x) \phi(x) \mathrm{d} x
$$

so that

$$
T(0, \cdot)=T^{i n}
$$

\section{Step 7: Identification of the limiting system}

In Steps 4 and 5, we have proved that

$$
\frac{\mathrm{d}}{\mathrm{d} t} \int_{\Omega} T(t, x) \Phi(x) \mathrm{d} x+\sigma \int_{\Omega} \nabla_{x} T(t, x) \cdot \nabla \Phi(x) \mathrm{d} x+4 \pi \sigma \int_{\Omega}(\varrho(x) T(t, x)-\vartheta(t, x)) \Phi(x) \mathrm{d} x=0
$$

and

$$
\frac{\mathrm{d}}{\mathrm{d} t}\left(\int_{\Omega} T(t, x) \Phi(x) \mathrm{d} x+\frac{\sigma}{\sigma^{\prime}} \int_{\Omega} \vartheta(t, x) \Phi(x) \mathrm{d} x\right)+\sigma \int_{\Omega} \nabla_{x} T(t, x) \cdot \nabla \Phi(x) \mathrm{d} x=0
$$

for each $\Phi \in C_{c}^{1}(\bar{\Omega})$. By linear combination, one finds that

$$
\begin{array}{r}
\frac{\mathrm{d}}{\mathrm{d} t} \int_{\Omega} T(t, x) \phi(x) \mathrm{d} x+\sigma \int_{\Omega} \nabla_{x} T(t, x) \cdot \nabla \phi(x) \mathrm{d} x+4 \pi \sigma \int_{\Omega}(\varrho(x) T(t, x)-\vartheta(t, x)) \phi(x) \mathrm{d} x=0, \\
\frac{\mathrm{d}}{\mathrm{d} t} \int_{\Omega} \vartheta(t, x) \psi(x) \mathrm{d} x+4 \pi \sigma^{\prime} \int_{\Omega}(\vartheta(t, x)-\varrho(x) T(t, x)) \psi(x) \mathrm{d} x=0,
\end{array}
$$

for all $\phi, \psi \in C_{c}^{1}(\bar{\Omega})$. Since $T, \vartheta \in C_{b}\left([0,+\infty) ; L^{2}(\Omega)\right)$ and $T \in L^{2}\left(0, \tau ; H^{1}(\Omega)\right)$ for each $\tau>0$, the identities above hold for all $\phi, \psi \in H^{1}(\Omega)$ by a straightforward density argument. 
Thus $(T, \vartheta)$ is the unique weak solution to $(2.11)$ with initial data $\left(T^{i n}, \vartheta^{i n}\right)$.

By compactness, this implies that

$$
T_{\epsilon} \rightarrow T \text { in } L^{\infty}\left([0,+\infty) ; L^{2}(\Omega)\right) \text { weak-* and in } L^{2}\left(0, \tau ; H^{1}(\Omega)\right) \text { weak }
$$

while

$$
\vartheta_{\epsilon} \rightarrow \vartheta \text { in } L^{\infty}\left([0,+\infty) ; L^{2}(\Omega)\right) \text { weak-* }
$$

without extracting subsequences.

\section{Proof of Lemmas $6.1,6.2,6.3$ and 6.4}

When $\psi=1$, the solution to the boundary value problem (6.2) is given by

$$
\chi[1](z)=\frac{\epsilon r_{\epsilon}}{r_{\epsilon}-\epsilon}\left(\frac{1}{|z|}-\frac{1}{r_{\epsilon}}\right) \mathbf{1}_{B\left(0, r_{\epsilon}\right) \backslash B(0, \epsilon)}(z)+\mathbf{1}_{B(0, \epsilon)}(z)
$$

for all $z \in \mathbf{R}^{3}$. In that case

$$
\|\chi[1]\|_{L^{2}\left(\mathbf{R}^{3}\right)}^{2}=\frac{4 \pi}{3} \epsilon^{2} r_{\epsilon}, \quad\|\nabla \chi[1]\|_{L^{2}\left(\mathbf{R}^{3}\right)}^{2}=4 \pi \frac{\epsilon r_{\epsilon}}{r_{\epsilon}-\epsilon} \sim 4 \pi \epsilon \quad \text { as } \epsilon \rightarrow 0 .
$$

Proof of Lemma 6.1. First, by the maximum principle and the mean value theorem, one has

$$
\begin{aligned}
\left\|\chi\left[\phi\left(x_{i}+\cdot\right)-\phi\left(x_{i}\right)\right]\right\|_{L^{\infty}\left(\mathbf{R}^{3}\right)} & \leq\left\|\phi\left(x_{i}+\cdot\right)-\phi\left(x_{i}\right)\right\|_{L^{\infty}\left(\mathbf{R}^{3}\right)} \\
& \leq \min \left(2\|\phi\|_{L^{\infty}\left(\mathbf{R}^{3}\right)},\|\nabla \phi\|_{L^{\infty}\left(\mathbf{R}^{3}\right)} \epsilon\right) .
\end{aligned}
$$

Since the functions $x \mapsto \chi\left[\phi\left(x_{i}+\cdot\right)-\phi\left(x_{i}\right)\right]\left(x-x_{i}\right)$ have disjoint supports by (2.18), one has both

$$
\left\|\mathcal{Q}_{\epsilon}\right\|_{L^{\infty}(\Omega)} \leq \sup _{1 \leq i \leq N}\left\|\chi\left[\phi\left(x_{i}+\cdot\right)-\phi\left(x_{i}\right)\right]\right\|_{L^{\infty}\left(\mathbf{R}^{3}\right)} \leq 2\|\phi\|_{L^{\infty}\left(\mathbf{R}^{3}\right)},
$$

and

$$
\begin{aligned}
\left\|\mathcal{Q}_{\epsilon}\right\|_{L^{2}(\Omega)}^{2} & \leq \sum_{i=1}^{N}\left\|\chi\left[\phi\left(x_{i}+\cdot\right)-\phi\left(x_{i}\right)\right]\right\|_{L^{2}\left(\mathbf{R}^{3}\right)}^{2} \\
& \leq N \mid B\left(0, r_{\epsilon}\right)\|\| \chi\left[\phi\left(x_{i}+\cdot\right)-\phi\left(x_{i}\right)\right] \|_{L^{\infty}\left(\mathbf{R}^{3}\right)}^{2} \\
& \leq N \cdot \frac{4}{3} \pi r_{\epsilon}^{3}\|\nabla \phi\|_{L^{\infty}\left(\mathbf{R}^{3}\right)}^{2} \epsilon^{2}=\frac{4}{3} \pi\|\nabla \phi\|_{L^{\infty}\left(\mathbf{R}^{3}\right)}^{2} \epsilon^{2} \rightarrow 0
\end{aligned}
$$

as $\epsilon \rightarrow 0$. Next

$$
\begin{aligned}
\left\|\nabla \chi\left[\phi\left(x_{i}+\cdot\right)-\phi\left(x_{i}\right)\right]\right\|_{L^{2}\left(\mathbf{R}^{3}\right)}^{2}= & \left\|\nabla \chi\left[\phi\left(x_{i}+\cdot\right)-\phi\left(x_{i}\right)\right]\right\|_{L^{2}(B(0, \epsilon))}^{2} \\
& +\left\|\nabla \chi\left[\phi\left(x_{i}+\cdot\right)-\phi\left(x_{i}\right)\right]\right\|_{L^{2}\left(B\left(0, r_{\epsilon}\right) \backslash B(0, \epsilon)\right)}^{2} .
\end{aligned}
$$

First

$$
\begin{aligned}
\left\|\nabla \chi\left[\phi\left(x_{i}+\cdot\right)-\phi\left(x_{i}\right)\right]\right\|_{L^{2}(B(0, \epsilon))}^{2} & \leq \frac{4}{3} \pi \epsilon^{3}\left\|\nabla \chi\left[\phi\left(x_{i}+\cdot\right)-\phi\left(x_{i}\right)\right]\right\|_{L^{\infty}(B(0, \epsilon))}^{2} \\
& =\frac{4}{3} \pi \epsilon^{3}\|\nabla \phi\|_{L^{\infty}(B(0, \epsilon))}^{2} .
\end{aligned}
$$

Since $\chi\left[\phi\left(x_{i}+\cdot\right)-\phi\left(x_{i}\right)\right]$ is a harmonic function on $B\left(0, r_{\epsilon}\right) \backslash B(0, \epsilon)$, it minimizes the Dirichlet integral among functions with the same boundary values. Thus

$$
\left\|\nabla \chi\left[\phi\left(x_{i}+\cdot\right)-\phi\left(x_{i}\right)\right]\right\|_{L^{2}\left(B\left(0, r_{\epsilon}\right) \backslash B(0, \epsilon)\right)}^{2} \leq\left\|\nabla \chi_{i, \epsilon}\right\|_{L^{2}\left(B\left(0, r_{\epsilon}\right) \backslash B(0, \epsilon)\right)}^{2}
$$


where

$$
\chi_{i, \epsilon}(z)=\left(\phi\left(x_{i}+\epsilon \frac{z}{|z|}\right)-\phi\left(x_{i}\right)\right) \frac{r_{\epsilon}-|z|}{r_{\epsilon}-\epsilon} .
$$

Straightforward computations show that

$$
\begin{aligned}
\nabla \chi_{i, \epsilon}(z)= & \left(I-\frac{z \otimes z}{|z|^{2}}\right) \nabla \phi\left(x_{i}+\epsilon \frac{z}{|z|}\right) \frac{\epsilon}{|z|} \frac{r_{\epsilon}-|z|}{r_{\epsilon}-\epsilon} \\
& -\left(\phi\left(x_{i}+\epsilon \frac{z}{|z|}\right)-\phi\left(x_{i}\right)\right) \frac{1}{r_{\epsilon}-\epsilon} \frac{z}{|z|}
\end{aligned}
$$

so that

$$
\begin{aligned}
\left|\nabla \chi_{i, \epsilon}(z)\right|^{2} \leq & \left|\nabla \phi\left(x_{i}+\epsilon \frac{z}{|z|}\right)\right|^{2} \frac{\epsilon^{2}}{|z|^{2}} \frac{\left(r_{\epsilon}-|z|\right)^{2}}{\left(r_{\epsilon}-\epsilon\right)^{2}} \\
& +\left(\phi\left(x_{i}+\epsilon \frac{z}{|z|}\right)-\phi\left(x_{i}\right)\right)^{2} \frac{1}{\left(r_{\epsilon}-\epsilon\right)^{2}} .
\end{aligned}
$$

Thus

$$
\left\|\nabla \chi_{i, \epsilon}\right\|_{L^{2}\left(B\left(0, r_{\epsilon}\right) \backslash B(0, \epsilon)\right)}^{2} \leq \frac{8 \pi}{3}\|\nabla \phi\|_{L^{\infty}\left(\mathbf{R}^{3}\right)}^{2} \epsilon^{2} r_{\epsilon}+O\left(\epsilon^{3} r_{\epsilon}\right)
$$

so that

$$
\begin{aligned}
\left\|\nabla \mathcal{Q}_{\epsilon}\right\|_{L^{2}(\Omega)}^{2} & \leq \sum_{i=1}^{N}\left\|\nabla \chi\left[\phi\left(x_{i}+\cdot\right)-\phi\left(x_{i}\right)\right]\right\|_{L^{2}\left(\mathbf{R}^{3}\right)}^{2} \\
& \leq \frac{4 \pi}{3}\|\nabla \phi\|_{L^{\infty}\left(\mathbf{R}^{3}\right)}^{2} N\left(\epsilon^{3}+2 \epsilon^{2} r_{\epsilon}\right) \\
& =\frac{4 \pi}{3}\|\nabla \phi\|_{L^{\infty}\left(\mathbf{R}^{3}\right)}^{2}\left(\epsilon^{2}+2 \epsilon r_{\epsilon}\right) \rightarrow 0
\end{aligned}
$$

as $\epsilon \rightarrow 0$. Hence $\mathcal{Q}_{\epsilon} \rightarrow 0$ in $H^{1}(\Omega)$ strong as $\epsilon \rightarrow 0$.

Proof of Lemma 6.2. Assume that $0<\epsilon<\frac{1}{8}$. Since the functions $x \mapsto \chi[1]\left(x-x_{i}\right)$ have disjoint supports by (2.18), one has

$$
\begin{aligned}
& \left\|\left.\mathcal{S}_{\epsilon}(t, \cdot)\right|_{L^{2}(\Omega)} ^{2} \leq \sum_{i=1}^{N} T_{i, \epsilon}(t)^{2}\right\| \chi[1] \|_{L^{2}\left(\mathbf{R}^{3}\right)}^{2} \\
& \left\|\nabla \mathcal{S}_{\epsilon}(t, \cdot)\right\|_{L^{2}(\Omega)}^{2} \leq \sum_{i=1}^{N} T_{i, \epsilon}(t)^{2}\|\nabla \chi[1]\|_{L^{2}\left(\mathbf{R}^{3}\right)}^{2} .
\end{aligned}
$$

Thus

$$
\left\|\mathcal{S}_{\epsilon}(t, \cdot)\right\|_{L^{2}(\Omega)}^{2} \leq \frac{4 \pi}{3} \epsilon^{2} r_{\epsilon} \sum_{i=1}^{N} T_{i, \epsilon}(t)^{2} \leq \frac{4 \pi}{3} \frac{\sigma^{\prime}}{\sigma} C^{i n} \epsilon r_{\epsilon} \rightarrow 0
$$

as $\epsilon \rightarrow 0$, while

$$
\begin{aligned}
\left\|\nabla \mathcal{S}_{\epsilon}(t, \cdot)\right\|_{L^{2}(\Omega)}^{2} & \leq 4 \pi \frac{\epsilon r_{\epsilon}}{r_{\epsilon}-\epsilon} \sum_{i=1}^{N} T_{i, \epsilon}(t)^{2} \\
& \leq 8 \pi \epsilon \sum_{i=1}^{N} T_{i, \epsilon}(t)^{2} \leq 8 \pi \frac{\sigma^{\prime}}{\sigma} C^{i n} .
\end{aligned}
$$

Hence $\mathcal{S}_{\epsilon}(t, \cdot) \rightarrow 0$ in $H^{1}(\Omega)$ weak, uniformly in $t \geq 0$ as $\epsilon \rightarrow 0$. 
Now for $\mathcal{R}_{\epsilon}$. First

$$
\begin{aligned}
\left\|\mathcal{R}_{\epsilon}\right\|_{L^{2}(\Omega)}^{2} & =\sum_{i=1}^{N}\left\|\chi\left[\phi\left(x_{i}\right)\right]\right\|_{L^{2}\left(\mathbf{R}^{3}\right)}^{2} \\
& \leq \sum_{i=1}^{N} \frac{4}{3} \pi \phi\left(x_{i}\right)^{2} \epsilon^{2} r_{\epsilon} \leq \frac{4}{3} \pi\|\phi\|_{L^{\infty}\left(\mathbf{R}^{3}\right)}^{2} \epsilon r_{\epsilon} \rightarrow 0
\end{aligned}
$$

as $\epsilon \rightarrow 0$, because the functions $x \mapsto \chi[1]\left(x-x_{i}\right)$ have disjoint supports by (2.18). By the same token

$$
\begin{aligned}
\left\|\nabla \mathcal{R}_{\epsilon}\right\|_{L^{2}(\Omega)}^{2} & =\sum_{i=1}^{N}\left\|\nabla \chi\left[\phi\left(x_{i}\right)\right]\right\|_{L^{2}\left(\mathbf{R}^{3}\right)}^{2} \\
& \leq \sum_{i=1}^{N} 4 \pi \phi\left(x_{i}\right)^{2} \frac{\epsilon r_{\epsilon}}{r_{\epsilon}-\epsilon} \leq 4 \pi\|\phi\|_{L^{\infty}\left(\mathbf{R}^{3}\right)}^{2} \frac{N \epsilon r_{\epsilon}}{r_{\epsilon}-\epsilon}=O(1)
\end{aligned}
$$

as $\epsilon \rightarrow 0$. Thus $\mathcal{R}_{\epsilon} \rightarrow 0$ in $H^{1}(\Omega)$ weak as $\epsilon \rightarrow 0$.

Proof of Lemma 6.3. One has

$$
\begin{aligned}
\sum_{i=1}^{N} \int_{B\left(0, r_{\epsilon}\right) \backslash B(0, \epsilon)} \nabla \chi\left[T_{i, \epsilon}(t)\right](z) \cdot \nabla \chi\left[\phi\left(x_{i}\right)\right](z) \mathrm{d} z & =\|\nabla \chi[1]\|_{L^{2}\left(\mathbf{R}^{N}\right)}^{2} \sum_{i=1}^{N} T_{i, \epsilon}(t) \phi\left(x_{i}\right) \\
& =4 \pi \frac{\epsilon r_{\epsilon}}{r_{\epsilon}-\epsilon} \sum_{i=1}^{N} T_{i, \epsilon}(t) \phi\left(x_{i}\right) \\
& =4 \pi \frac{r_{\epsilon}}{r_{\epsilon}-\epsilon} \iint_{\Omega \times \mathbf{R}} \phi(x) \theta \mu_{\epsilon}(t, \mathrm{~d} x \mathrm{~d} \theta) \\
& \rightarrow 4 \pi \iint_{\Omega \times \mathbf{R}} \phi(x) \theta \mu(t, \mathrm{~d} x \mathrm{~d} \theta)=4 \pi \int_{\Omega} \phi(x) \vartheta(t, x) \mathrm{d} x
\end{aligned}
$$

as $\epsilon \rightarrow 0$.

Proof of Lemma 6.4. First

$$
\begin{aligned}
\sum_{i=1}^{N} \frac{\partial}{\partial n}\left(\chi\left[\phi\left(x_{i}\right)\right]\left(x-x_{i}\right)\right) \delta_{\partial B\left(x_{i}, r_{\epsilon}\right)} & =\sum_{i=1}^{N} \phi\left(x_{i}\right) \frac{x-x_{i}}{\left|x-x_{i}\right|} \cdot \nabla \chi[1]\left(x-x_{i}\right) \delta_{\partial B\left(x_{i}, r_{\epsilon}\right)} \\
& =-\sum_{i=1}^{N} \phi\left(x_{i}\right) \frac{\epsilon r_{\epsilon}}{r_{\epsilon}-\epsilon} \frac{1}{\left|x-x_{i}\right|^{2}} \delta_{\partial B\left(x_{i}, r_{\epsilon}\right)} \\
& =-\frac{\epsilon r_{\epsilon}}{r_{\epsilon}^{2}\left(r_{\epsilon}-\epsilon\right)} \sum_{i=1}^{N} \phi\left(x_{i}\right) \delta_{\partial B\left(x_{i}, r_{\epsilon}\right) .}
\end{aligned}
$$

Next we recall that

$$
\sum_{i=1}^{N} \phi\left(x_{i}\right) r_{\epsilon} \delta_{\partial B\left(x_{i}, r_{\epsilon}\right)} \rightarrow 4 \pi \varrho \phi \quad \text { strongly in } H^{-1}\left(\mathbf{R}^{3}\right)
$$

as $\epsilon \rightarrow 0$. This result has been proved by Cioranescu-Murat [8] in the case where $x_{i}$ are distributed periodically; see formula (64) and Appendix 1 in [9] for a proof adapted to the setting of the present paper.

With the explicit formula above and the fact that $\frac{\epsilon}{r_{\epsilon}^{2}\left(r_{\epsilon}-\epsilon\right)} \rightarrow 1$ as $\epsilon \rightarrow 0$, this concludes the Proof of Lemma 6.4. 


\section{Conclusion}

We have established the homogenization limit for the heat equation in a composite material consisting of a background medium with small spherical inclusions of another material with infinite thermal conductivity. The asymptotic distribution of inclusions is an arbitrary absolutely continuous positive probability density $\varrho$. Our analysis requires that the distance between neighboring inclusions remains larger than a certain threshold which scales as $N^{-1 / 3}$, where $N$ is the number of inclusions, while the size of the inclusions scales as $N^{-1}$. The homogenized model is a system that consists of a heat equation for the temperature in the background medium, coupled to an ordinary differential equation for the temperature in the inclusions. The coupling terms are local, linear and do not involve derivatives of the unknowns; their strength is proportional to the density of inclusions $\varrho$.

Extending our result to the case of an unbounded domain $\Omega$ should be straightforward, but would require some modifications in our arguments. For instance, it may be necessary to drop the assumption that $1 / \varrho$ is bounded on $\Omega$, and to assume instead that

$$
\frac{1}{N} \sum_{i=1}^{N}\left|x_{i}\right|^{2} \leq C^{i n} \quad \text { for all } N \geq 1
$$

for some positive constant $C^{i n}$. The definition of the inner product $(\cdot \mid \cdot)_{\mathcal{V}}$ in Section 3 should be modified accordingly.

A more interesting open problem would be to obtain an equation for the spatial distribution of temperature in the inclusions under exactly the same scaling assumptions as in Theorem 2.6. This could be relevant to the modeling of sprays. With the notations of the proof of Theorem 2.6, the question would be to investigate the evolution in the time variable $t$ of limit points of

$$
\mu_{\epsilon}(t, \mathrm{~d} x \mathrm{~d} \theta):=\frac{1}{N} \sum_{i=1}^{N} \delta_{x_{i}} \otimes \delta_{T_{i, \epsilon}(t)}
$$

as $\epsilon \rightarrow 0$ in the weak topology of probability measures. The analysis in the present work leads to a closed system governing the evolution of the averaged quantities

$$
\varrho=\int \mu(\mathrm{d} \theta) \quad \text { and } \vartheta=\int \theta \mu(\mathrm{d} \theta)
$$

if $\mu$ is a weak limit point of some subsequence of $\mu_{\epsilon}$ as $\epsilon \rightarrow 0$. Obtaining an equation for $\mu$ itself seems more complicated.

We conclude this section with a remark of a more technical nature on the arguments used in the proof the homogenization theorem (Thm. 2.6). Specifically, we draw the reader's attention to the difference between the two classes of test functions used in this proof. Cioranescu and Murat have stressed the importance of the condition $\left.\Theta_{\epsilon}\right|_{\partial B\left(x_{i}, \epsilon\right)}=0$ in obtaining the limit (6.4): see the discussion of assumptions (H5) and (H5') on p. 108 in [8]. This accounts for the different arguments leading to the damping terms in the homogenized equations for $T$ and for $\theta$ - in other words, to the difference between the proofs of (6.3) and (6.4), or equivalently between the proofs of Lemmas 6.3 and 6.4 .

\section{Appendix A. Some lemmas on Evolution EQuations}

Let $\mathcal{V}$ and $\mathcal{H}$ be two separable Hilbert spaces such that $\mathcal{V} \subset \mathcal{H}$ with continuous inclusion and $\mathcal{V}$ is dense in $\mathcal{H}$. The Hilbert space $\mathcal{H}$ is identified with its dual and the map

$$
\mathcal{H} \ni u \mapsto L_{u} \in \mathcal{V}^{\prime}
$$


where $L_{u}$ is the linear functional

$$
L_{u}: \mathcal{V} \ni v \mapsto(u \mid v)_{\mathcal{H}} \in \mathbf{R},
$$

identifies $\mathcal{H}$ with a dense subspace of $\mathcal{V}^{\prime}$.

Lemma A.1. Assume that

$$
v \in L^{2}(0, T ; \mathcal{V}) \quad \text { and } \frac{d L_{v}}{\mathrm{~d} t} \in L^{2}\left(0, T ; \mathcal{V}^{\prime}\right)
$$

Then

(a) the function $v$ is a.e. equal to a unique element of $C([0, T], \mathcal{H})$ still denoted $v$;

(b) this function $v \in C([0, T], \mathcal{H})$ satisfies

$$
\frac{1}{2}\left|v\left(t_{2}\right)\right|_{\mathcal{H}}^{2}-\frac{1}{2}\left|v\left(t_{1}\right)\right|_{\mathcal{H}}^{2}=\int_{t_{1}}^{t_{2}}\left\langle\frac{\mathrm{d} L_{v}}{\mathrm{~d} t}(t), v(t)\right\rangle_{\mathcal{V}^{\prime}, \mathcal{V}} \mathrm{d} t
$$

for all $t_{1}, t_{2} \in[0, T]$.

Statement (a) follows from Proposition 2.1 and Theorem 3.1 in Chapter 1 of [12], and statement (b) from Theorem II.5.12 of [5].

Lemma A.2. Let $L \in L^{2}\left(0, T ; \mathcal{V}^{\prime}\right)$ satisfy

$$
\langle L(t), w\rangle_{\mathcal{V}^{\prime}, \mathcal{V}}=0 \text { for a.e. } t \in[0, T]
$$

for all $w \in \mathcal{V}$. Then

$$
L(t)=0 \text { for a.e. } t \in[0, T] .
$$

Proof. Pick $\mathcal{N}_{w} \subset[0, T]$ negligible such that $L$ is defined on $[0, T] \backslash \mathcal{N}_{w}$ and

$$
\langle L(t), w\rangle_{\mathcal{V}^{\prime}, \mathcal{V}}=0 \text { for all } t \in[0, T] \backslash \mathcal{N}_{w} .
$$

Let $\mathcal{D}$ be a dense countable subset of $\mathcal{V}$ and let

$$
\overline{\mathcal{N}}:=\bigcup_{w \in \mathcal{D}} \mathcal{N}_{w}
$$

For all $t \in[0, T] \backslash \overline{\mathcal{N}}$, one has

$$
\langle L(t), w\rangle_{\mathcal{V}^{\prime}, \mathcal{V}}=0 \text { for all } w \in \mathcal{D} \quad \text { so that } L(t)=0
$$

because $L(t)$ is a continuous linear functional on $\mathcal{V}$ and $\mathcal{D}$ is dense in $\mathcal{V}$.

The next lemma recalls the functional background for Green's formula in the context of evolution equations.

Lemma A.3. Let $\Omega$ be an open subset of $\mathbf{R}^{N}$ with smooth boundary, and let $T>0$. Denote by $n$ the unit outward normal field on $\partial \Omega$. Let $\rho \in C\left([0, T] ; L^{2}(\Omega)\right)$ and $m \in L^{2}\left((0, T) \times \Omega, \mathbf{R}^{N}\right)$. Assume that

$$
\partial_{t} \rho+\operatorname{div}_{x} m=0 \quad \text { in the sense of distributions in }(0, T) \times \Omega .
$$

Then

(a) the vector field $m$ has a normal trace $\left.m \cdot n\right|_{(0, T) \times \partial \Omega} \in H_{00}^{1 / 2}((0, T) \times \partial \Omega)^{\prime}$; 
(b) for each $\psi \in H^{1}(\Omega)$

$$
\frac{\mathrm{d}}{\mathrm{d} t} \int_{\Omega} \rho(\cdot, x) \psi(x) \mathrm{d} x-\int_{\Omega} m(\cdot, x) \cdot \nabla_{x} \psi(x) \mathrm{d} x=-\left\langle\left. m \cdot n\right|_{\partial \Omega},\left.\psi\right|_{\partial \Omega}\right\rangle_{H^{-1 / 2}(\partial \Omega), H^{1 / 2}(\partial \Omega)}
$$

in $H^{-1}(0, T)$.

Proof. Let $\chi \in C_{c}^{\infty}(\mathbf{R})$ be such that

$$
\chi(t)=1 \text { for } t \in[-1, T+1] \quad \text { and } \operatorname{supp}(\chi) \subset[-2, T+2]
$$

Define

$$
\bar{\rho}(t, x):= \begin{cases}\rho(t, x) & \text { if } 0 \leq t \leq T \\ \chi(t) \rho(0, x) & \text { if } t<0 \\ \chi(t) \rho(T, x) & \text { if } t>T\end{cases}
$$

and

$$
\bar{m}(t, x):= \begin{cases}m(t, x) & \text { if } 0 \leq t \leq T \\ 0 & \text { if } t \notin[0, T]\end{cases}
$$

so that the vector field $X:=(\bar{\rho}, \bar{m})$ is an extension of $(\rho, m)$ to $\mathbf{R} \times \Omega$ satisfying

$$
X \in L^{2}\left(\mathbf{R} \times \Omega ; \mathbf{R}^{N+1}\right) .
$$

Besides

$$
\left(\partial_{t} \bar{\rho}+\operatorname{div}_{x} \bar{m}\right)(t, x)=\chi^{\prime}(t)\left(\mathbf{1}_{t<0} \rho(0, x)+\mathbf{1}_{t>T} \rho(T, x)\right)=: S(t, x)
$$

with $S \in L^{2}(\mathbf{R} \times \Omega)$ so that

$$
\operatorname{div}_{t, x} X=S \in L^{2}(\mathbf{R} \times \Omega) .
$$

Therefore $X$ has a normal trace on the boundary $\partial(\mathbf{R} \times \Omega)=\mathbf{R} \times \partial \Omega$, denoted $\left.X \cdot n\right|_{\mathbf{R} \times \partial \Omega} \in H^{-1 / 2}(\mathbf{R} \times \partial \Omega)$.

Let $\phi \in H_{00}^{1 / 2}((0, T) \times \partial \Omega)$; denote by $\bar{\phi}$ its extension by 0 to $\mathbf{R} \times \partial \Omega$. Thus $\bar{\phi} \in H^{1 / 2}(\mathbf{R} \times \partial \Omega)$ and there exists $\bar{\Phi} \in H^{1}(\mathbf{R} \times \Omega)$ such that $\bar{\phi}=\left.\bar{\Phi}\right|_{\mathbf{R} \times \partial \Omega}$. The normal trace of $m$ is then defined as follows: by Green's formula

$$
\begin{aligned}
\left\langle\left. m \cdot n\right|_{\mathbf{R} \times \partial \Omega}, \phi\right\rangle_{H_{00}^{1 / 2}((0, T) \times \partial \Omega)^{\prime}, H_{00}^{1 / 2}((0, T) \times \partial \Omega)} & :=\left\langle\left. X \cdot n\right|_{\mathbf{R} \times \partial \Omega}, \bar{\phi}\right\rangle_{H^{1 / 2}(\mathbf{R} \times \partial \Omega)^{\prime}, H^{1 / 2}(\mathbf{R} \times \partial \Omega)} \\
& =\iint_{\mathbf{R} \times \Omega}\left(\bar{\rho} \partial_{t} \bar{\Phi}+\bar{m} \cdot \nabla_{x} \bar{\Phi}+S \bar{\Phi}\right)(t, x) \mathrm{d} x \mathrm{~d} t .
\end{aligned}
$$

Applying Green's formula on $(0, T) \times \Omega$ shows that two different extensions of the vector field $(\rho, m)$ define the same distribution $\left.m \cdot n\right|_{(0, T) \times \partial \Omega}$ on $(0, T) \times \partial \Omega$. This completes the proof of statement (a). 
As for statement (b), let $\kappa \in H_{0}^{1}(0, T)$ and $\psi \in H^{1}(\Omega)$, define $\Phi(t, x):=\kappa(t) \psi(x)$ and let $\bar{\Phi}$ be the extension of $\Phi$ by 0 to $\mathbf{R} \times \Omega$, so that $\bar{\Phi} \in H^{1}(\mathbf{R} \times \Omega)$. Thus $\phi=\left.\Phi\right|_{(0, T) \times \partial \Omega} \in H_{00}^{1 / 2}((0, T) \times \partial \Omega)$ and

$$
\begin{aligned}
\left\langle\left\langle\left. m \cdot n\right|_{\partial \Omega},\left.\psi\right|_{\partial \Omega}\right\rangle_{H^{-1 / 2}(\partial \Omega), H^{1 / 2}(\partial \Omega)}, \kappa\right\rangle_{H^{-1}(0, T), H_{0}^{1}(0, T)} & =\left\langle\left. m \cdot n\right|_{(0, T) \times \partial \Omega}, \phi\right\rangle_{H_{00}^{1 / 2}((0, T) \times \partial \Omega)^{\prime}, H_{00}^{1 / 2}((0, T) \times \partial \Omega)} \\
& =\iint_{\mathbf{R} \times \Omega}\left(\bar{\rho} \partial_{t} \bar{\Phi}+\bar{m} \cdot \nabla_{x} \bar{\Phi}+S \bar{\Phi}\right)(t, x) \mathrm{d} x \mathrm{~d} t \\
& =\int_{0}^{T} \int_{\Omega}\left(\rho(t, x) \kappa^{\prime}(t) \psi(x)+m(t, x) \cdot \nabla \psi(x) \kappa(t)\right) \mathrm{d} x \mathrm{~d} t \\
= & -\left\langle\frac{\mathrm{d}}{\mathrm{d} t} \int_{\Omega} \rho(t, x) \psi(x) \mathrm{d} x, \kappa\right\rangle_{H^{-1}(0, T), H_{0}^{1}(0, T)} \\
& +\int_{0}^{T} \int_{\Omega} m(t, x) \cdot \nabla \psi(x) \kappa(t) \mathrm{d} x \mathrm{~d} t
\end{aligned}
$$

which is precisely the identity in statement (b).

Acknowledgements. We thank Philippe Villedieu for suggesting the problem of deriving the temperature equations for multiphase flows in thermal local non-equilibrium. V.R. acknowledges the support by the GNFM (research project 2011: "Analisi di modelli per sistemi a scala multipla"). This work has been partially supported by the French national research agency (ANR) grant ANR-10-BLAN-1119-02 SAMOVAR.

\section{REFERENCES}

[1] G. Bal, Transport through diffusive and nondiffusive regions, embedded objects and clear layers. SIAM J. Appl. Math. 62 (2002) 1677-1697.

[2] M. Bellieud, Homogenization of evolution problems for a composite medium with very small and heavy inclusions. ESAIM: COCV 11 (2005) 266-284.

[3] M. Bellieud, A notion of capacity related to elasticity. Applications to homogenization. Arch. Rational Mech. Anal. 203 (2012) $137-187$.

[4] M. Bellieud, C. Licht and S. Orankitjaroen, Nonlinear capacitary problems for a non periodic distribution of fibers. Appl. Math. Res. Express 2014 (2014) 1-51.

[5] F. Boyer and P. Fabrie, Éléments d'analyse pour l'étude de quelques modèles d'écoulements de fluides visqueux incompressibles. Math. Appl., vol. 52. Springer Verlag, Berlin, Heidelberg (2006).

[6] C.E. Brennen, Fundamentals of Multiphase Flows. Cambridge University Press (2005).

[7] H. Brezis, Analyse Fonctionnelle. Théorie et Applications. Masson, Paris (1987).

[8] D. Cioranescu and F. Murat, Un terme étrange venu d'ailleurs. In Nonlinear Partial Differential Equations and their Applications, Collège de France Seminar vol. II, Paris 1979-1980; vol. 60 of Res. Notes Math. Pitman, Boston, London (1982) 98-138.

[9] L. Desvillettes, F. Golse and V. Ricci, The Mean-Field Limit for Solid Particles in a Navier-Stokes Flow. J. Stat. Phys. 131 (2008) 941-967.

[10] F. Fichot, F. Duval, N. Trégourès, C. Béchaud and M. Quintard, The impact of thermal non-equilibrium and large-scale 2D/3D effects on debris bed reflooding and coolability. Nucl. Eng. Design 236 (2006) 2144-2163.

[11] V.A. L'vov and E. Ya. Hruslov, Perturbations of a viscous incompressible fluid by small particles. Theor. Appl. Quest. Differ. Equ. Algebra 267 (1978) 173-177.

[12] J.-L. Lions and E. Magenes, Problèmes aux limites non homogènes et applications, vol. 1. Dunod, Paris (1968).

[13] A. Mikelic and M. Primicerio, Homogenization of heat conduction in materials with periodic inclusions of a perfect conductor. In Progress in partial differential equations: calculus of variations, applications. Pont-Mousson, 1991, vol. 267 of Pitman Res. Notes Math. Ser. Longman Sci. Tech., Harlow (1992) 244-256.

[14] A. Mikelic, M. Primicerio, Homogenization of the heat equation for a domain with a network of pipes with a well-mixed fluid. Ann. Mat. Pura Appl. 166 (1994) 227-251.

[15] F. Petit, F. Fichot, M. Quintard, Ecoulement diphasique en milieu poreux: modèle à non-équilibre local. Int. J. Therm. Sci. 38 (1999) 239-249.

[16] P.-A. Raviart and J.-M. Thomas, Introduction à l'analyse numérique des équations aux dérivées partielles. Masson, Paris, 1983.

[17] J. Wloka, Partial Differential Equations. Cambridge University Press, Cambridge (1987). 